\title{
Apoptosis resistance of senescent cells is an intrinsic barrier for senolysis induced by cardiac glycosides
}

\author{
Pavel I. Deryabin ${ }^{1} \cdot$ Alla N. Shatrova ${ }^{2} \cdot$ Aleksandra V. Borodkina $^{1}$ (i)
}

Received: 15 July 2021 / Revised: 24 September 2021 / Accepted: 13 October 2021 / Published online: 29 October 2021

(c) The Author(s) 2021

\begin{abstract}
Targeted elimination of senescent cells, senolysis, is one of the core trends in the anti-aging therapy. Cardiac glycosides were recently proved to be a broad-spectrum senolytics. Here we tested senolytic properties of cardiac glycosides towards human mesenchymal stem cells (hMSCs). Cardiac glycosides had no senolytic ability towards senescent hMSCs of various origins. Using biological and bioinformatic approaches we compared senescence development in 'cardiac glycosides-sensitive' A549 and '-insensitive' hMSCs. The absence of senolysis was found to be mediated by the effective potassium import and increased apoptosis resistance in senescent hMSCs. Weakening "antiapoptotic defense" predisposes hMSCs to senolysis. We revealed that apoptosis resistance, previously recognized as a common characteristic of senescence, in fact, is not a general feature of senescent cells. Moreover, only apoptosis-prone senescent cells are sensitive to cardiac glycosides-induced senolysis. Thus, we can speculate that the effectiveness of senolysis might depend on whether senescent cells indeed become apoptosis-resistant as compared to their proliferating counterparts.
\end{abstract}

\section{Graphic abstract}
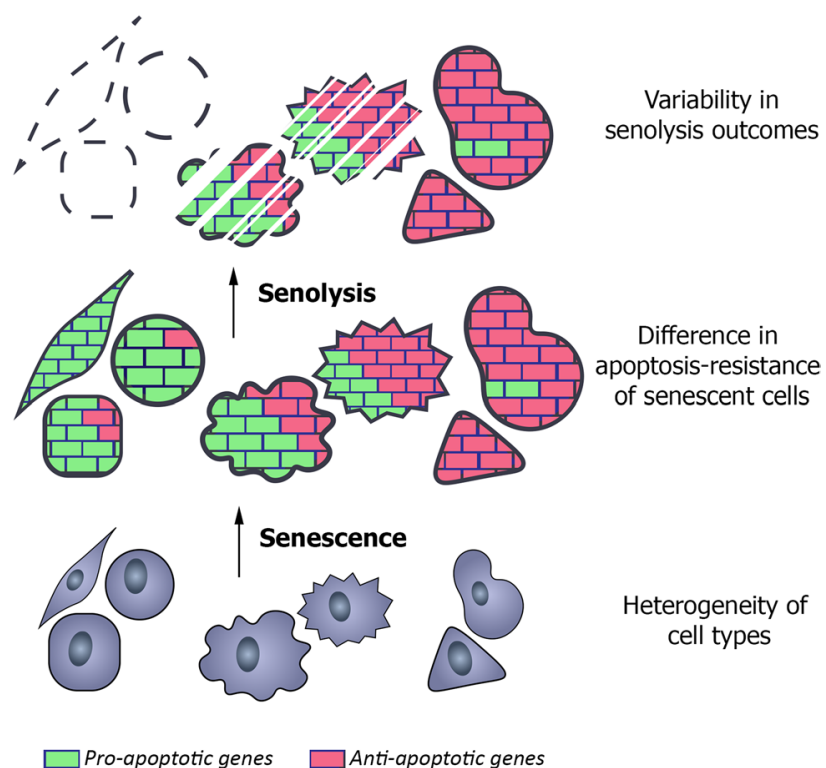

Heterogeneity of cell types

Keywords Stem cells $\cdot$ Senolysis $\cdot$ Senescence $\cdot$ Apoptosis $\cdot$ Stress resistance

Aleksandra V. Borodkina

borodkina618@gmail.com

Extended author information available on the last page of the article

\section{Abbreviations}

hMSCs Human mesenchymal stem cells

SASP Senescence-associated secretory phenotype

SA- $\beta$-Gal $\quad$ Senescence-associated $\beta$-galactosidase 
END-MSCs Human MSCs derived from endometrium

AD-MSCs

Human MSCs derived from adipose tissue

DP-MSCs

Human MSCs derived from dental pulp

WJ-MSCs

DEGs

GSEA
Differentially expressed genes

Gene set enrichment analysis

\section{Introduction}

Today cellular senescence is considered as a common reaction of almost all types of proliferating cells, including cancer and stem ones, as well as some post-mitotic cells to a variety of stressful stimuli [1-3]. The following types of cellular senescence can be distinguished accordingly to the origin of the inducing factor: replicative (due to the DNA damage at the shortened telomere ends), oncogene-induced (in response to aberrant activation of oncogenic signaling), stress-induced (mediated by the DNA damage caused by the oxidative stress, heat shock, UV and $\gamma$ radiation, etc.) and chemotherapy-induced (activated in cancer cells in response to chemotherapeutic drugs) [4-7]. There is heterogeneity in markers expressed by senescent cells depending on both cell type and an insult used to induce senescence. However, there are several common features typical for the most types of senescent cells. The essential characteristic of senescence for any kind of dividing cells is the irreversible proliferation loss [8]. The irreversibility of the cell cycle arrest is controlled by the cyclin-dependent kinase (CDK) inhibitors p16 and p21 and is often regulated by the tumor suppressor protein p53 [8, 9]. The other important features of senescent cells are the activation of a persistent DNA damage response; cell hypertrophy, which often arises as a result of impaired ribosomal biogenesis and protein synthesis; disturbance of lysosomal degradation and dysfunction of the rest degradation systems; increased activity of the specific lysosomal enzyme senescence-associated- $\beta$-galactosidase; various mitochondrial alterations; acquisition of the senescence-associated secretory phenotype (SASP), composed of pro-inflammatory factors, matrix degrading enzymes, reactive oxygen species, etc.; epigenetic and chromatin landscape alterations, including formation of senescence-associated heterochromatic foci and senescence-associated distention of satellites [8-10]. In other words, senescent cells preserve metabolic activity and vitality, but their functioning is significantly altered comparing to the origin cells.

It is now clear that senescent cells can modify surrounding microenvironment affecting both neighboring cells and cellular niches, what may lead to tissue malfunctioning and therefore may be related to the progression of aging and agerelated diseases $[11,12]$. Keeping that in mind, today more attention is focused on the strategies for targeted "killing" of senescent cells [13-15]. To this end, a novel class of drugs

termed senolytics is actively developing. Senolytics target signaling pathways that contribute to the resistance of senescent cells towards apoptosis, thus inducing apoptosis preferentially in senescent cells [16]. The list of senolytics is constantly replenishing with the new agents. The most known compounds with the stated senolytic activity are navitoclax (Bcl-2 family inhibitor), combination of dasatinib (an inhibitor of multiple tyrosine kinases) and quercetin (a natural flavonol), Hsp90 inhibitors, MDM2 inhibitors, FOXO4-p53 interfering peptide, a BET family protein degrader, uPARspecific CAR-T, galacto-conjugated navitoclax and various senolytic natural compounds [16-24]. Recently, using highthroughput drug screening two independent research groups identified cardiac glycosides, particularly ouabain, digoxin and bufalin, as a broad-spectrum senolytics [25, 26]. It is worth mentioning that almost all of the declared senolytics have limitations, such as undesirable side-effects or ineffectiveness towards some cell types.

Mesenchymal stem cells (MSCs), found virtually in all postnatal organs/tissues, are characterized by the capacity to self-renew (symmetric divisions) and to differentiate, thus contributing to maintenance and regeneration of the residing tissue [27]. Due to these unique properties, along with the potent anti-inflammatory and immunosuppressive functions, MSCs are broadly applied in the cell replacement therapy for treatment of various diseases, including diabetes mellitus, multiple sclerosis, myocardial infarction and so on [28]. However, similar to their differentiated progenies and other unipotent cells, MSCs are able to senesce either replicatively or prematurely in response to oncogenes' activation and stressful stimuli [29, 30]. MSCs' senescence has a plenty of undesirable aftermaths, among which are exhaustion of the pool of stem cells, reduced tissue maintenance and regeneration, impaired differentiation capacity, SASP-mediated senescence spreading, reduced angiogenic properties, modification of stem cell niche [29, 31-33]. Taking into account biological significance of the proper MSCs' functioning, senescent MSCs might be considered as the crucial target for senolysis. In line with this suggestion, a number of reviews highlighting possible advantageous outcomes of senescent MSCs removal has been published over the past year [29, $31,34,35]$. Nevertheless, there are only few experimental data regarding this issue [36-40].

Within the present study, we demonstrate for the first time that cardiac glycosides, namely ouabain and bufalin, fail to display senolytic activity towards human MSCs (hMSCs) derived from endometrium (END-MSCs), adipose tissue (AD-MSCs), dental pulp (DP-MSCs) and Warton jelly (WJ-MSCs). In addition, we confirm that both cardiac glycosides are able to induce apoptosis preferentially in senescent A549 and SK-HEP-1, as it was previously described in the pilot studies $[25,26]$. By assessing alterations in ionic homeostasis caused by the $\mathrm{Na}^{+} / \mathrm{K}^{+}$-ATPase blocking and 
expression levels of the related genes we reveal that the absence of ouabain-induced senolysis might be mediated by the enhanced effectiveness of the compensatory $\mathrm{K}^{+}$import in senescent END-MSCs as compared to senescent A549. Furthermore, using advanced bioinformatics we demonstrate that senescence of END-MSCs, resistant to ouabain-induced senolysis, is accompanied by the acquisition of apoptosisresistant phenotype, while senescence of ouabain-sensitive A549 is not. Importantly, blocking activity of antiapoptotic protein MCL-1 prior to cardiac glycoside treatment resulted in senolysis in apoptosis-resistant senescent END-MSCs. Therefore, we provide clear evidence that apoptosis-resistance is not a general feature of senescent cells. Based on the data obtained we conclude that the effectiveness of senolytic approaches might depend on whether cells indeed became apoptosis-resistant during senescence development.

\section{Materials and methods}

\section{Cells}

END-MSCs, WJ-MSCs, DP-MSCs, AD-MSCs, A549 and SK-Hep1 were obtained from the Russian Collection of Cell Cultures (Institute of Cytology, Saint-Petersburg, Russia). A549 and SK-Hep1 lines were authenticated by karyology, tumorigenicity, isoenzyme (LDH and G6PD) tests and STR analysis. Cells were cultured in complete medium DMEM/ F12 (Gibco BRL) supplemented with 10\% FBS (HyClone), $1 \%$ penicillin-streptomycin (Gibco BRL) and $1 \%$ glutamax (Gibco BRL). All cells were routinely tested for mycoplasma contamination.

\section{Senescence- and stress-inducing conditions}

For oxidative stress-induced senescence END-MSCs/WJMSCs were treated with $200 \mu \mathrm{M} / 100 \mu \mathrm{M} \mathrm{H}_{2} \mathrm{O}_{2}$ (Sigma) for $1 \mathrm{~h}$. For doxorubicin-induced senescence DP-MSCs/A549 were treated with $1 \mu \mathrm{M}$ of doxorubicin (Veropharm) for 3 days. In each case cells were considered senescent not earlier than 14 days after treatment. For replicative senescence AD-MSCs earlier than 4th passage were identified as control cells and later than 10th as senescent ones. For etoposide-induced senescence A549/END-MSCs/SK-Hep1 were treated with $2 \mu \mathrm{M} / 5 \mu \mathrm{M} / 3 \mu \mathrm{M}$ etoposide (Veropharm) for 3 days and analyzed not earlier than 7 days after senescence induction. In this case, treatment design completely coincided with those described in the study from which RNA-seq data originated (Wang et al., 2017). Two cardiac glycosides we applied as senolytic compounds-Ouabain (Sigma) and Bufalin (Calbiochem).

In order to compare stress-resistance between control and senescent END-MSCs or A549, cells were treated either with $400 / 800 \mu \mathrm{M} \mathrm{H}_{2} \mathrm{O}_{2}$ (Sigma) for $1 \mathrm{~h}$ or with $0.3 / 1 \mu \mathrm{M}$ staurosporine (Sigma). Cell viability was analyzed in $48 \mathrm{~h}$ after stress induction.

To block MCL-1 activity END-MSCs were pretreated with $10 \mu \mathrm{M}$ of A-1210477 (Sigma) for 3 days.

\section{Flow cytometry analysis}

Measurements of cell viability, proliferation, cell size, autofluorescence, apoptosis rates, caspase 3/7 cleavage, mitochondrial membrane potential, membrane depolarization were carried out by flow cytometry. Flow cytometry was performed using the CytoFLEX (Beckman Coulter) and the obtained data were analyzed using CytExpert software version 2.0. Adherent cells were rinsed twice with PBS and harvested by trypsinization. Detached cells were pooled and resuspended in fresh medium and then counted and analyzed for autofluorescence. In order to access cell viability, $50 \mu \mathrm{g} / \mathrm{ml}$ propidium iodide (Life Technologies) was added to each sample just before analysis. The cell size was evaluated by cytometric forward light scattering of PI-negative cells. Apoptosis induction was verified using Annexin-V-APC (Invitrogen) and DAPI (Sigma) co-staining following manufactures instructions. Caspase activity was assessed using CellEvent ${ }^{\mathrm{TM}}$ Caspase-3/7 Green Flow Cytometry Assay Kit (Invitrogen) following manufactures protocol. Loss of mitochondrial membrane potential was assessed using the ratiometric dye JC-1 (Invitrogen). The staining procedure was carried out in accordance with the manufacture's protocol. For membrane depolarization we used DiBAC4(3) fluorescent probe (Invitrogen).

\section{Senescence-associated $\beta$-galactosidase staining}

Senescence-associated $\beta$-Galactosidase (SA- $\beta$-Gal) staining was performed using senescence $\beta$-galactosidase staining kit (Cell Signaling Technology) according to manufacturer's instructions. Quantitative analysis of images was produced with the application of MatLab package, according to the algorithm described previously [41]. For each experimental point not less 50 randomly selected cells were analyzed.

\section{Western blotting}

Western blotting was performed as described previously [41]. SDS-PAGE electrophoresis, transfer to nitrocellulose membrane and immunoblotting with ECL (Thermo Scientific) detection were performed according to standard manufacturer's protocols (Bio-Rad Laboratories). Antibodies against the following proteins were used: glyceraldehyde3-phosphate dehydrogenase (GAPDH) (clone 14C10), phospho-p53 (Ser15), p21 (clone 12D1), phospho-Rb (Ser807/811), HMGB1 (clone D3E5), as well as horseradish 
peroxidase-conjugated goat antirabbit IgG. Dilution rates were 1:1000 for all primary antibodies and 1:7000 for secondary ones. All antibodies were purchased from Cell Signaling. The Scion Image 4.0 (Scion Corporation) was used to select and determine the background-subtracted density of the bands in all the gels and blots.

\section{Transcriptomic analysis}

Samples from the following three Gene Expression Omnibus (GEO) RNA-seq datasets were used in the analysis: GSE102639 (GSM2742113-GSM2742114 and GSM2742121-GSM2742122 for control and senescent A549 cells, accordingly); GSE122081 (GSM3454482-GSM3454484 and GSM3454500-GSM3454502 for control and senescent IMR-90 cells, accordingly); and our dataset GSE160702 (GSM4877895-GSM4877898 and GSM4877907-GSM4877910 for control and senescent END-MSCs, respectively). The data for all the datasets were processed in the same way.

Raw reads data underwent quality filtering and adapter trimming via FilterByTile and BBDuk scripts from the BBtools package (version 38.75) using the default options. The remaining reads were additionally filtered and trimmed with the use of trimFilter script from the FastqPuri package (version 1.0.7). Trimming operation was applied for both ends of reads if they contained N's or their quality was below the quality threshold set to 27 , all reads shorter than 25 bases were discarded. The quality control of trimming was held with the FastQC software (version 0.11.7) and FastqPuri scripts. The reads, having passed all operations, comprised no less than $90 \%$ of the initial data.

Transcript abundances were estimated using the Salmon lightweight mapping (version 1.1.0) running in the selective alignment mode. The list of decoys was generated based on the Gencode human reference genome GRCh38.p13 (release 33) and used further for building the index on concatenated transcriptome and genome Gencode reference files (release 33) using k-mer size of 21. Mapping operations were run with additional flags-numBootstraps 30-seqBiasgcBias-validateMappings. Resulting mapping rates were around $70 \%$.

Further data processing was performed using $\mathrm{R}$ version 3.6.3 with the Tidyverse collection of packages (version 1.3.0). Estimated gene counts, metadata and transcript ranges were loaded into $\mathrm{R}$ and summarized to a gene level using tximeta (version 1.4.5). Resulting count matrix was filtered to contain rows having at least 5 estimated counts across all samples, the resulting matrix contained 20,400 genes.

For the PCA and heatmap representation the read counts were normalized using rlog transformation from the DESeq2 package (version 1.26.0). Heatmaps were constructed with the use of genefilter (version 1.38.0) and pheatmap (version
1.0.12) R packages. Validation of division samples by senescence variable was conducted via subsetting normalized read counts by genes related to the Gene Ontology term "Cellular senescence" (GO 0090398). Differential expression analysis and log fold changes estimation were computed using DESeq2 for the last variable in the design formula controlling for cells senescence status, ouabain treatment reaction and an interaction of the indicated factors. To strengthen differential expression testing, log fold changes correction using combination of adaptive shrinkage estimator from the apeglm package (version 1.8.0) and specifying additional log fold change threshold equal to 0.667 was applied. Resulting shrunken estimates were used further for gene ranking and running Gene Set Enrichment Analysis using clusterProfiler (version 3.14.3) and fgsea (version 1.12.0) R packages with $p$ values adjustment for multiple comparisons according to the Benjamin-Hochberg method.

Affymetrix microarray samples for control and senescent AD-MSCs were obtained from the GEO database (GSE66236) and processed with Phantasus web-application with $\log 2$ and quantile counts normalization. Gene Set Enrichment Analysis was performed with the use fgsea (version 1.12.0) R package.

\section{RNA extraction, reverse transcription and real time PCR}

RNA extraction, reverse transcription and real time PCR were performed as described in our previous study [32]. Reagents for RNA extraction (ExtractRNA reagent), for reverse transcription (MMLV RT kit) and for real time PCR (HS SYBR kit) were obtained from Evrogen. Gene expression levels were assessed using the Realtime PCR BioRad CFX-96 amplifier (BioRad) with the following running program for all investigated genes: 40 cycles of melting for $10 \mathrm{~s}$ at $95{ }^{\circ} \mathrm{C}$, annealing for $15 \mathrm{~s}$ at $57.5^{\circ} \mathrm{C}$ and synthesis for $15 \mathrm{~s}$ at $72{ }^{\circ} \mathrm{C}$. Melting curves analysis was applied to control specificity of reactions. The following analysis of the obtained data was performed using the Bio-Rad CFX Manager software (BioRad) with standard 2deltaCt quantification method with the use of GAPDH expression as the reference. Primer sequences are listed in Table 1.

\section{Statistical analysis}

To get significance in the difference between two groups Student's $t$ test or Welch's $t$ test was applied. For multiple comparisons between groups, ANOVA with Tukey's HSD was used. Unless otherwise indicated, all quantitative data are shown as mean \pm SD and the asterisks indicate significant differences as follows: ns, not significant, $* p<0.05$, ${ }^{* *} p<0.01, * * * p<0.001$. Statistical analysis was performed using R software. 
Table 1 Primer oligonucleotide sequences

\begin{tabular}{lll}
\hline$\#$ & Oligonucleotide & Sequence \\
\hline 1 & GAPDH forward & 5'-GAGGTCAATGAAGGGGTCAT-3' \\
2 & GAPDH reverse & 5'-AGTCAACGGATTTGGTCGTA-3' \\
3 & BAX forward & 5'-TGCTTCAGGGTTTCATCCA-3' \\
4 & BAX reverse & 5'-GGCGGCAATCATCCTCTG-3' \\
5 & KCNJ2 forward & 5'-TCCGAGGTCAACAGCTTCAC-3' \\
6 & KCNJ2 reverse & 5'-TTGGGCATTCATCCGTGACA-3' \\
7 & MCL1 forward & 5'-GATGATCCATGTTTTCAGCGAC-3' \\
8 & MCL1 reverse & 5'-CTCCACAAACCCATCCCAG-3' \\
9 & SLC12A forward & 5'-GAGGAGATGGACAGTAACCCC-3' \\
10 & SLC12A reverse & 5'-CTGGCTCAGGTTGGTGTAGTT-3' \\
11 & WNK4 forward & 5'-GTGAAGGCTGCGGAAGACTC-3' \\
12 & WNK4 reverse & 5'-CTGGGTCTCCATGTCCTCCTT-3' \\
\hline
\end{tabular}

\section{Results}

\section{$\mathrm{H}_{2} \mathrm{O}_{2}$-treated END-MSCs enter the premature senescence}

Within the present study, we used human mesenchymal stem cells isolated from desquamated endometrium (ENDMSCs), which satisfy the minimum criteria suggested by the ISCT for defining hMSCs [41]. Previously, we have developed the reliable experimental model to study various aspects of the premature senescence of END-MSCs [30]. Namely, we have shown that END-MSCs subjected to sublethal oxidative stress gradually acquired all the typical features of senescent cells, including persistent DNA damage foci and active DNA damage response, irreversible cell cycle arrest mediated by the classical $\mathrm{p} 53 / \mathrm{p} 21 / \mathrm{Rb}$ pathway, proliferation loss, cell hypertrophy, appearance of SA- $\beta-\mathrm{Gal}$ staining and development of senescence-associated secretory phenotype [30, 32, 42]. Here we applied the designed model to study senolytic effect of ouabain as well as to reveal the underlying intracellular alterations in ion homeostasis. Initially, by estimating the most common parameters we confirmed that single-dose $(1 \mathrm{~h}, 200 \mu \mathrm{M}) \mathrm{H}_{2} \mathrm{O}_{2}$ treatment was sufficient to induce senescence in END-MSCs. Importantly, stressed END-MSCs were considered senescent in two weeks after the oxidative stress; therefore, all the senescence markers were assessed not earlier than 14 days after $\mathrm{H}_{2} \mathrm{O}_{2}$ treatment. Indeed, $\mathrm{H}_{2} \mathrm{O}_{2}$ treatment of END-MSCs led to proliferation block, cell hypertrophy as indicated by the increased cell size, accumulation of the lipofuscin detected by the elevation of autofluorescence, appearance of the SA- $\beta$-Gal, activation of the $\mathrm{p} 21 / \mathrm{Rb}$ pathway and loss of HMGB1 together supporting senescence establishment under the chosen experimental conditions (Fig. 1a-c, e, f).

The most harmful effects of senescent cells at tissue and organismal levels are believed to be the consequence of their prolonged vitality. In line with this point, $\mathrm{H}_{2} \mathrm{O}_{2}$-treated END-MSCs retained high viability even at the late stages of senescence development (viability of senescent END-MSCs was assessed in 17 days (14 days +3 days) after the initial oxidative stress) (Fig. 1d). To sum up, sublethal $\mathrm{H}_{2} \mathrm{O}_{2}$-treatment of ENDMSCs is the appropriate model of the premature senescence and is relevant to investigate the effects of senolytic compounds on END-MSCs.

\section{Cardiac glycoside ouabain has no senolytic activity towards senescent END-MSCs in a wide concentration range}

Recent evidence suggests that cardiac glycosides, including ouabain, digoxin, bufalin, represent a family of compounds with senolytic activity $[25,26]$. Even though today cardiac glycosides are considered as the broad-spectrum senolytics, the data regarding their effects towards senescent hMSCs are lacking. Therefore, here we tested whether ouabain has the potential to induce death selectively in senescent ENDMSCs. To do so, we assessed viability of control and senescent END-MSCs after treatment with ouabain at the wide concentration range (from $10^{-7}$ to $10^{-5} \mathrm{M}$ ). Interestingly, neither concentration applied led to the noticeable decrease in the viability of both control and senescent cells on the first day after ouabain application (Fig. 2 and Supplemental Fig. S1).

However, on the third day after ouabain treatment we revealed significant dose-dependent decline in the viability of control END-MSCs (Fig. 2a and Supplemental Fig. S1). Unexpectedly, senescent cells turned out to be more resistant towards ouabain at each concentration tested. In line with this result, $10^{-6} \mathrm{M}$ ouabain led to more prone apoptotic death in control cells (20.75\% An+/PI- and $36.47 \% \mathrm{An}+/ \mathrm{PI}+)$ as compared to senescent ones (15.01\% An $+/ \mathrm{PI}-$ and $12.15 \%$ An+/PI+) (Fig. 2b). Obtained results clearly demonstrate that in context of senescent END-MSCs ouabain has no senolytic activity.

To exclude possible effects associated with variability of senescence-inducing stimuli on senolytic action of ouabain, we performed additional set of experiments. Namely, we treated END-MSCs with etoposide instead of oxidative stress to induce premature senescence. Etoposide-treated END-MSCs displayed all the features typical for senescent cells (Fig. 3a-e).

Importantly, ouabain had no senolytic action towards etoposide-treated senescent END-MSCs (Fig. 3f and Supplemental Fig. S2). These data provide additional confirmation for the above results and evidence that the lack of ouabaininduced senolysis is not the consequence of the concrete senescence trigger used to induce senescence. 

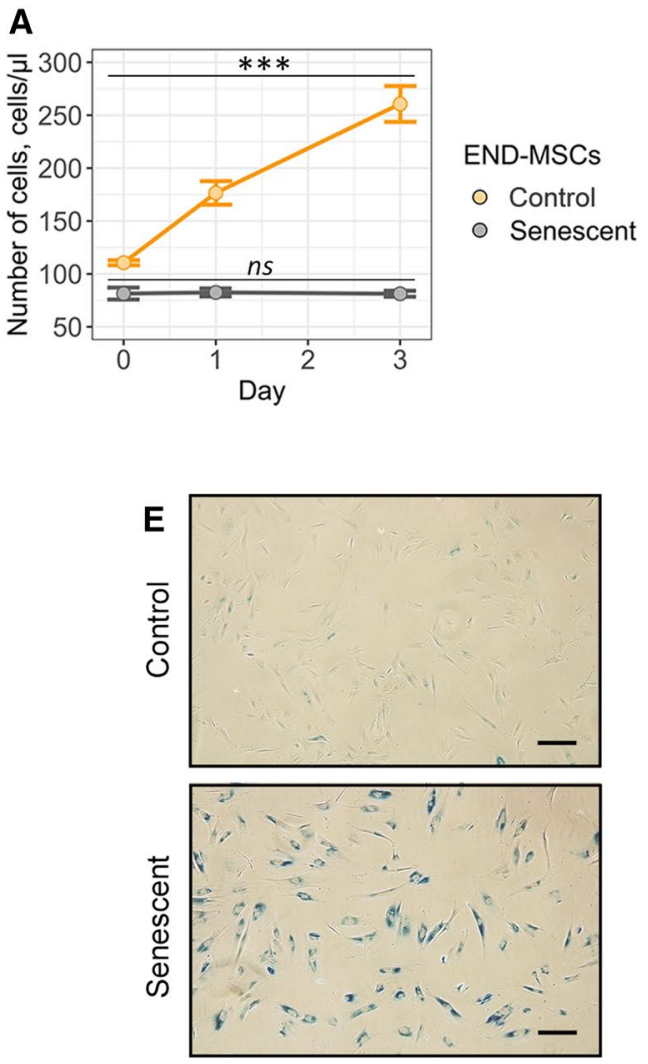

B
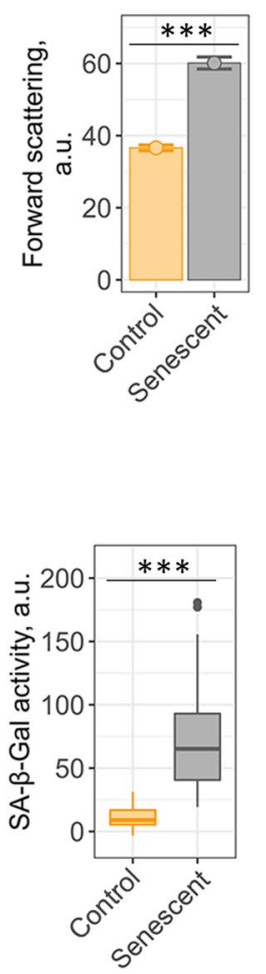

C

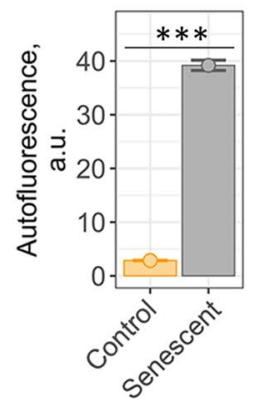

F

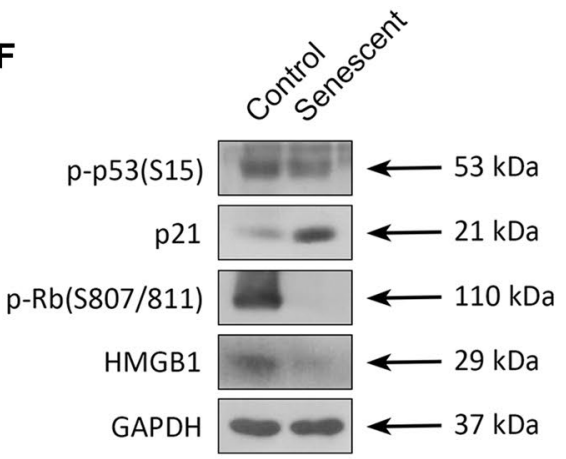

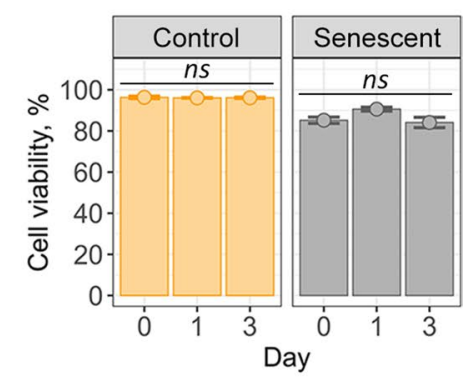

D 
A

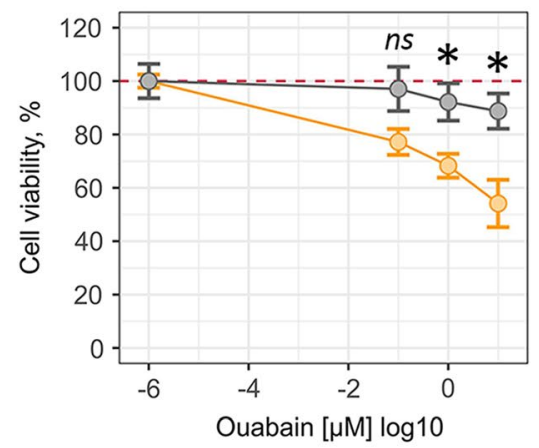

END-MSCs

O Control

○ Senescent
B

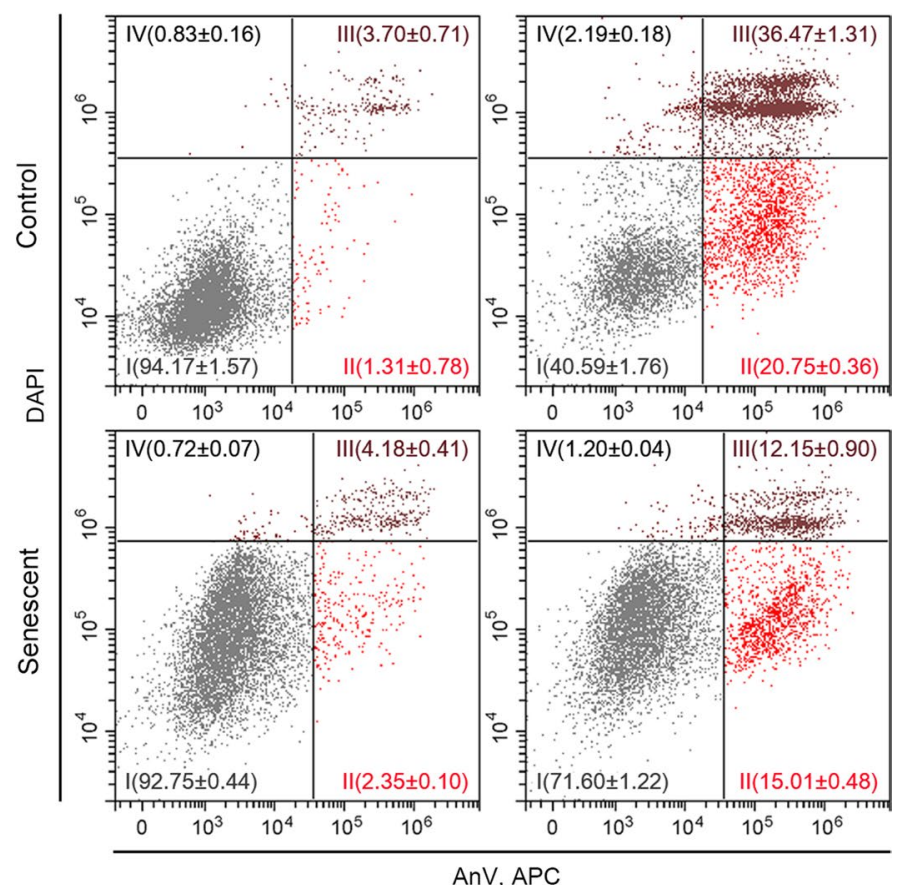

Fig. 2 Ouabain has no senolytic activity towards $\mathrm{H}_{2} \mathrm{O}_{2}$-treated senescent END-MSCs in a wide concentration range. a Relative cell viability (\%) of control and senescent END-MSCs in 3 days after treatment with $10^{-7}, 10^{-6}, 10^{-5} \mathrm{M}$ ouabain. b Apoptosis induction in END-

\section{Both cardiac glycosides ouabain and bufalin are able to induce cell death selectively in senescent A549 and SK-Hep1 cells}

Taking into account the fact that our results do not correspond with the recently published evidences regarding broad-spectrum senolytic action of cardiac glycosides, we decided to reproduce this effect using cellular model described in the relevant studies $[25,26]$. Thus, we performed series of experiments using control and senescent A549 lung carcinoma cells. Senescence in A549 cells was induced by etoposide treatment. Etoposide-induced senescence of A549 cells is a frequently used and thus well characterized model of therapy-induced senescence [43]. Also, ouabain was shown to selectively kill etoposide-treated senescent A549 cells [25]. To prove senescence in A549, we assessed proliferation rate, cell size, accumulation of lipofucine, SA- $\beta$-Gal staining, activation status of the p53/p21/ $\mathrm{Rb}$ pathway and expression level of HMGB1 (Fig. 6a-e).

To verify senolytic activity of ouabain towards senescent cancer cells, we first estimated dose-dependent cell viability. In line with our results described above, we were not able to detect any significant decline in the number of viable control or senescent A549 cells within $24 \mathrm{~h}$ after ouabain application (Supplemental Fig. S5a). However, in 3 days after treatment ouabain significantly reduced viability of senescent
MSCs upon $10^{-6} \mathrm{M}$ ouabain assessed by Annexin V/DAPI double staining. $n=3$ independent experiments. All data correspond to the mean \pm SD. Statistical significance was assessed by the Welch's $t$ test: $n s$ not significant, $* * * p<0.05$

A549 cells in a dose-dependent manner, while the number of control A549 cells decreased to a much lesser extent (Fig. 7a and Supplemental Fig. S5a). Namely, approximately $90 \%$ of control cells preserved viability at $10^{-6} \mathrm{M}$ ouabain compared to $50 \%$ of senescent A549 cells treated with the same dose (Fig. 7a). Moreover, senescent A549 cells were more prone towards bufalin-induced cell death than their control counterparts (Fig. 7b) (Fig. 5b and Supplemental Fig. S5b).

According to the published data, ouabain triggered caspase-3-dependent apoptosis in senescent A549 cells [26]. Indeed, we revealed significant increase in the double positive AnV/PI-fraction in senescent cancer cells treated with $10^{-6} \mathrm{M}$ ouabain (Fig. 7c). Furthermore, using fluorescent assay we observed activation of caspase- 3 in ouabain-treated senescent A549 cells (Fig. 7d). Taken together, these results are completely consistent with the data described by other authors and confirm senolytic activity of ouabain towards A549.

Also, we utilized doxorubicin instead of etoposide to cause senescence in A549 (Fig. 8a-e). As expected, doxorubicin-treated senescent A549, as well as etoposide-treated demonstrated higher sensitivity both to ouabain and bufalin compared to their control counterparts (Fig. $8 \mathrm{~g}$ and Supplemental Fig. S6). The latter confirms that senolytic effects of cardiac glycosides are independent of senescence inducing stimuli. Thus, cardiac glycosides indeed have senolytic 

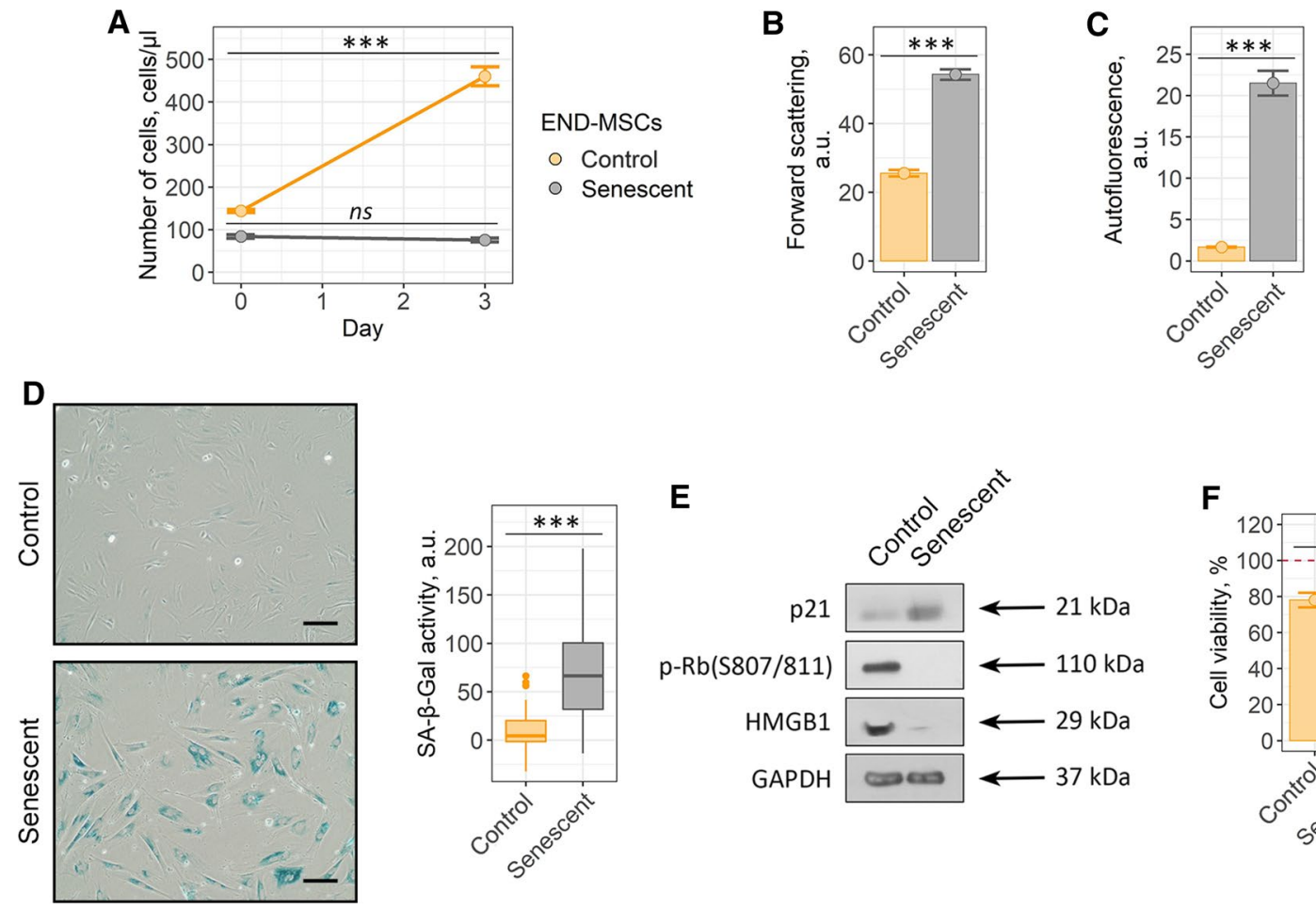

E
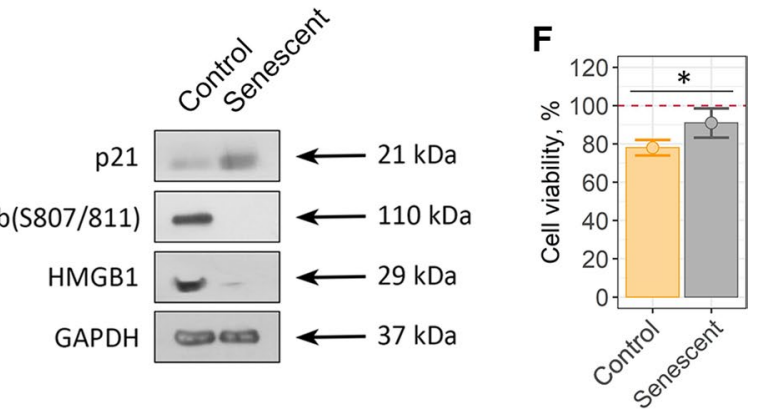

Fig. 3 Ouabain is unable to induce senolysis in etoposide-treated senescent END-MSCs. Validation of the etopisode-induced senescence model for END-MSCs: a proliferation ability, b cell size, $\mathbf{c}$ autofluorescence levels and d SA- $\beta$-Gal activity, e phosphorylation level of $\mathrm{Rb}$ and expression levels of $\mathrm{p} 21$ and HMGB1 proteins. $\mathbf{f}$ Relative cell viability (\%) of control and senescent cells after treat-

activity towards A549, but these compounds turned to be ineffective for targeted death induction in senescent hMSCs.

Finally, we decided to reproduce senolysis experiments on etoposide-treated liver cancer cells SK-Hep1, another cell model with proved senolytic effects of cardiac glycosides according to Guerrero's et al. study [25] (Fig. 9a-e). Etoposide-treated senescent SK-Hep1 demonstrated higher sensitivity to both agents compared to their control counterparts, proving for the senolytic action of cardiac glycosides towards this cell type (Fig. 9f and Supplemental Fig. S7).

Together these data evidence that selectivity of senolysis induced by cardiac glycosides relies more on cell nature than on the concrete senescence trigger.

\section{Disturbance of $\mathrm{K}^{+} / \mathrm{Na}^{+}$homeostasis caused by ouabain leads to different physiological reactions in control and senescent A549 and END-MSCs}

Having established the fact that senolytic effect of ouabain is cell type-dependent, further we tried to elucidate what may underlie the revealed differences in responses of hMSCs and A549. To this end we analyzed oxidative stressinduced senescence of END-MSCs and etoposide-induced ment with $10^{-6}$ ouabain. Values are mean \pm SD. For multiple groups comparisons at a one-way ANOVA was applied, $n=3, n s$ not significant, $* * * p<0.001$. For pair comparisons at b, c, d, f Welch's $t$ test was used, $n=3$ for $\mathbf{b}, \mathbf{c}, \mathbf{f} n=50$ for $\mathbf{d}, n s$ not significant, $* p<0.05$, $* * * p<0.001$. Scale bars for images are $500 \mu \mathrm{m}$. GAPDH was used as loading control

senescence of A549 more precisely. The main molecular mechanism of action of various cardiac glycosides is inhibition of $\mathrm{Na}^{+} / \mathrm{K}^{+}$-ATPase [44]. $\mathrm{Na}^{+} / \mathrm{K}^{+}$-ATPase is a plasma membrane enzyme that pumps sodium out of the cell while pumping potassium into the cell against concentration gradients and thus participating in maintenance $\mathrm{K}^{+}$and $\mathrm{Na}^{+}$ homeostasis. Therefore, we asked whether there might be any differences in basal and ouabain-induced ion homeostasis between control and senescent cells of two types.

The disturbance in $\mathrm{K}^{+}$and $\mathrm{Na}^{+}$homeostasis caused by ouabain should ultimately lead to the dissipation of the plasma membrane potential. Thus, further we used specific fluorescent probe DiBAC4(3) to measure transmembrane potential of nonexcitable cells. Increased fluorescence of this dye reflects membrane depolarization. In each cell type tested ouabain induced predictable membrane depolarization, confirming ionic disbalance (Fig. 10a). Notably, we observed comparable levels of membrane depolarization in control and senescent END-MSCs, while senescent A549 characterized by significantly more depolarized membrane compared to control A549 cells.

Other important consequences of ionic deregulation are changes in mitochondrial membrane potential (MMP). 

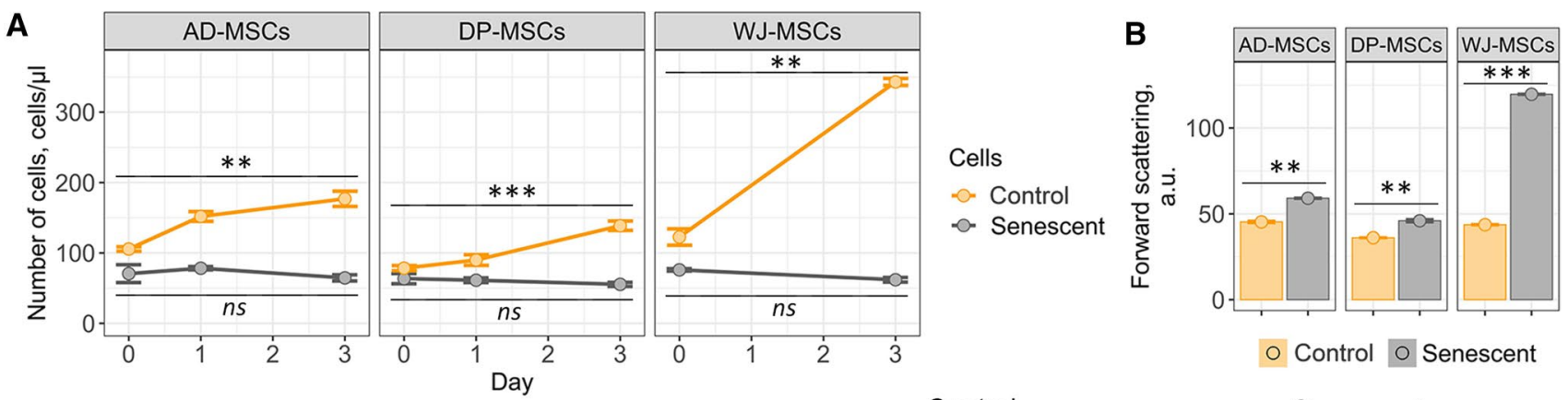

○ Control O Senescent

C

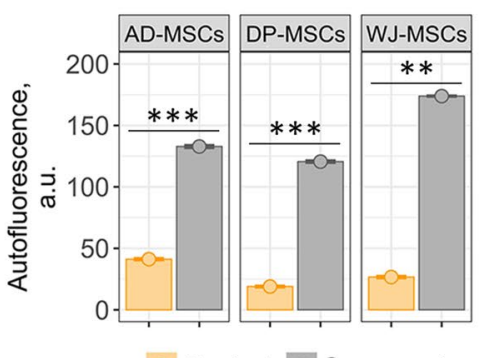

D
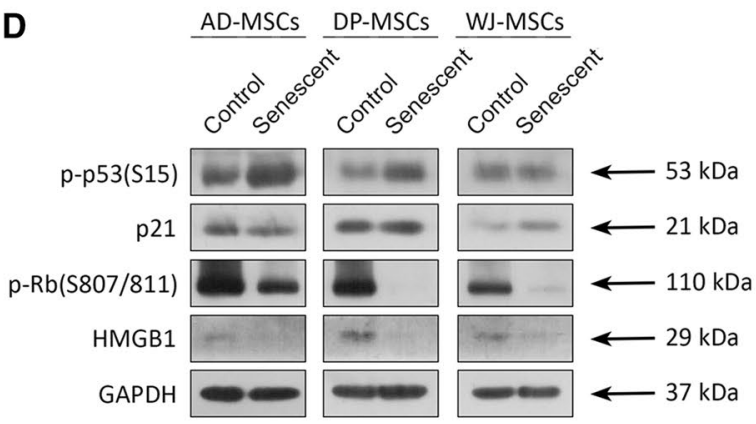

Control
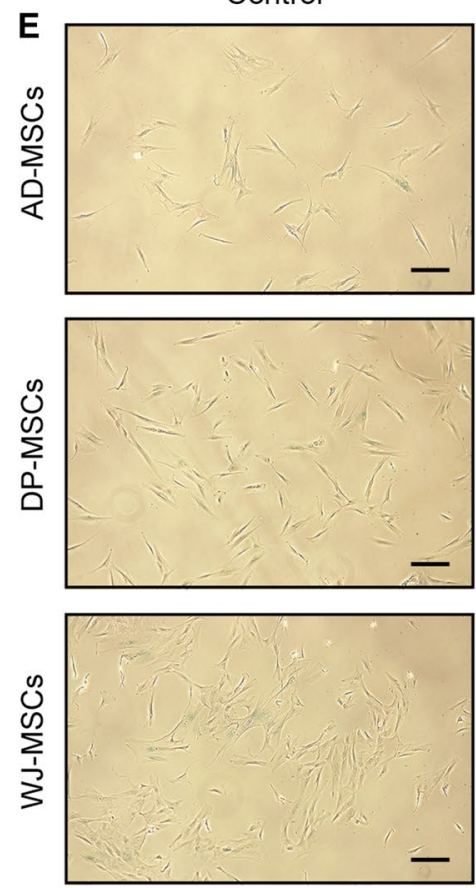
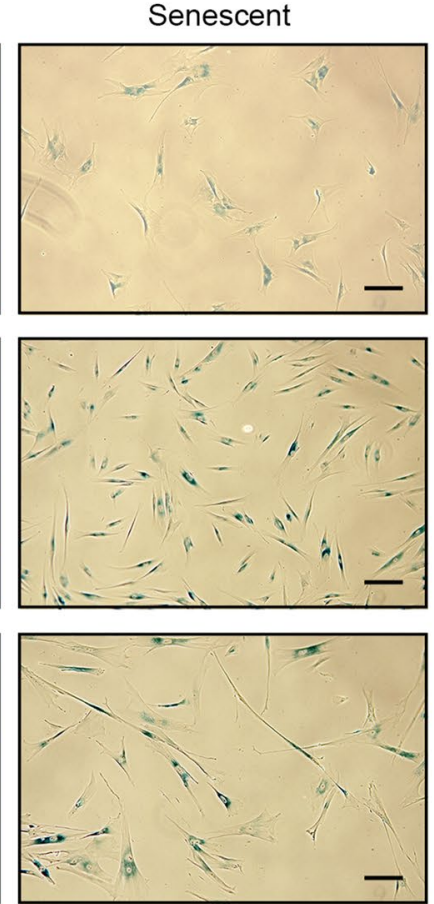

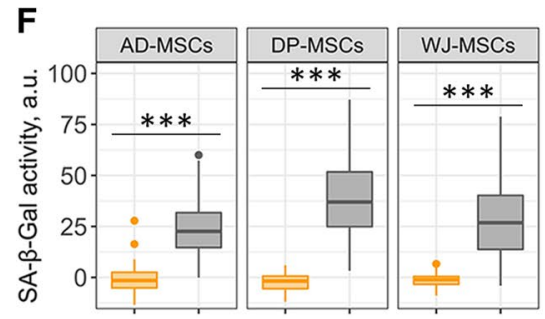

审Control 追Senescent

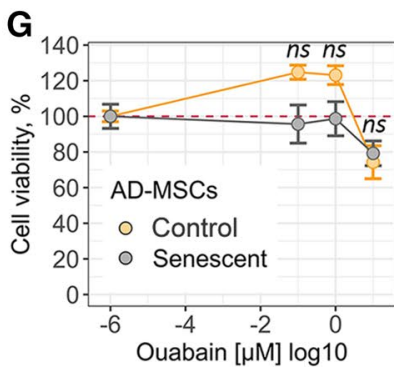

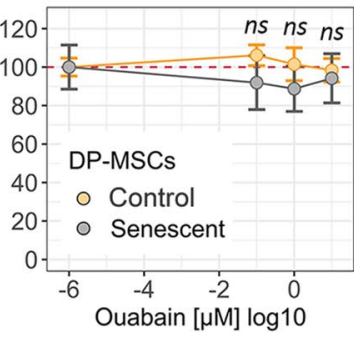

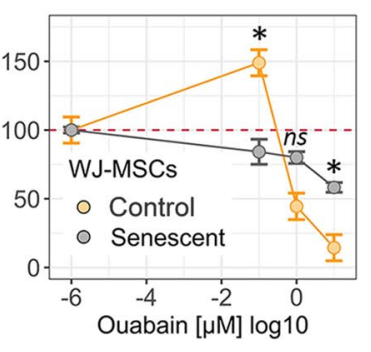

Fig. 4 Ouabain has no senolytic action towards AD-MSCs, DP-MSCs and WJ-MSCs. Validation of the three additional hMSCs senescence models, replicative for AD-MSCs and premature for DP-MSCs (doxorubicin induced) and WJ-MSCs (oxidative stress induced): a loss of proliferation ability and acquisition of elevated $\mathbf{b}$ cell size, $\mathbf{c}$ autofluorescence levels and $\mathbf{e}$ and $\mathbf{f}$ SA- $\beta$-Gal activity by senescent hMSCs as compared to the control cells. d Phosphorylation levels of p53 and $\mathrm{Rb}$ and expression levels of $\mathrm{p} 21$ and HMGB1 proteins in control and

Thus, using fluorescent probe JC-1 we assessed MMP in control and senescent cells upon ouabain treatment. It should be specifically highlighted that senescent cells are commonly characterized by decreased MMP reflecting senescent hMSCs. g Relative cell viability (\%) of control and senescent AD-MSCs, DP-MSCs and WJ-MSCs after treatment with $10^{-7}$, $10^{-6}, 10^{-5} \mathrm{M}$ ouabain, respectively. Values are mean $\pm \mathrm{SD}$. For multiple groups comparisons at an one-way ANOVA was applied, $n=3$, $n s$ not significant, ${ }^{*} p<0.01, * * * p<0.001$. For pair comparisons at $\mathbf{a}, \mathbf{b}, \mathbf{c}, \mathbf{e}$ and $\mathbf{g}$ Welch's $t$ test was used, $n=3$ for $\mathbf{a}, \mathbf{b}, \mathbf{c}, \mathbf{g} n=50$ for $\mathbf{f}$, $n s$ not significant, $* p<0.05, * * p<0.01, * * * p<0.001$. Scale bars for images are $500 \mu \mathrm{m}$. GAPDH was used as loading control

malfunctioning of mitochondria, alterations in energy metabolism and increased intracellular reactive oxygen species levels [45]. As expected, MMP in senescent ENDMSCs and A549 cells was lower than in the control ones, 

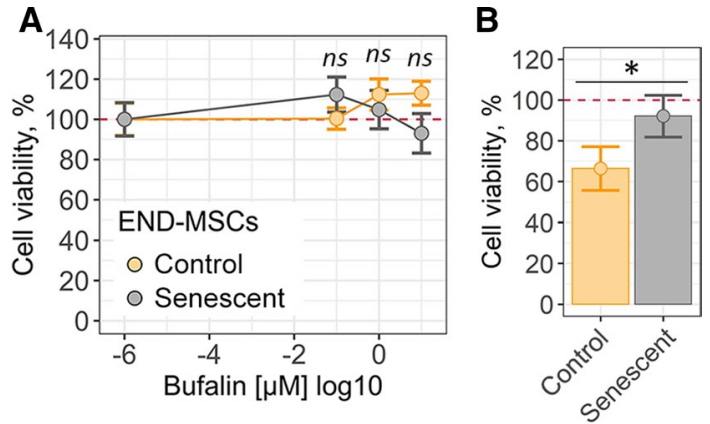

Fig. 5 Cardiac glycoside bufalin fails to kill senescent END-MSCs. a Relative cell viability (\%) of control and $\mathrm{H}_{2} \mathrm{O}_{2}$-treated senescent END-MSCs in 3 days after treatment with $10^{-7}, 10^{-6}, 10^{-5} \mathrm{M}$ bufalin. b Relative cell viability (\%) of control and etoposide-treated senescent END-MSCs after treatment with $10^{-6}$ bufalin. All data correspond to the mean \pm SD. Statistical significance was assessed by the Welch's $t$ test: $n s$ not significant, $* p<0.05$

though this decrease did not affect viability of senescent cells (Figs. 1d, 6a, 10b). Similar to the above results, ouabain induced pronounced MMP depolarization in senescent A549 compared to their proliferating counterparts, while effect on MMP of control and senescent END-MSCs was minimal (Fig. 10b).

Summarizing the results described within this part, we can conclude that blocking $\mathrm{Na}^{+} / \mathrm{K}^{+}$-ATPase by ouabain lead to the dramatic depolarization of plasma membrane and drop of MMP in case of senescent A549, contrarily to senescent END-MSCs.

\section{Alterations in the mechanisms of $\mathrm{K}^{+}$import mediate ouabain-resistance of senescent END-MSCs}

To clarify mechanisms mediating ouabain-induced changes in membrane polarization and MMP between senescent A549 and END-MSCs, further we employed advanced bioinformatic approach. The aim of the analysis was to reveal possible transcriptomic features mediating cell type dependent ouabain-resistance or -sensitivity of senescent cells. In other words, we asked the question: how senescence of END-MSCs (ouabain-resistant cells) differs from senescence of A549 (ouabaine-sensitive cells)? To answer this question, we utilized three independent RNA sequencing (RNA-Seq) datasets. The first one was RNA-Seq analysis
A
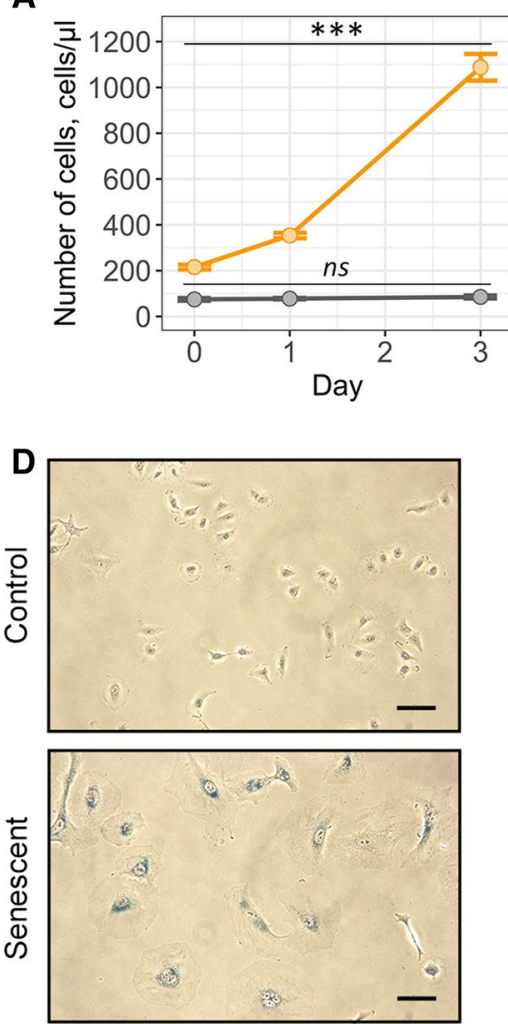

A549

O Control

O Senescent
B
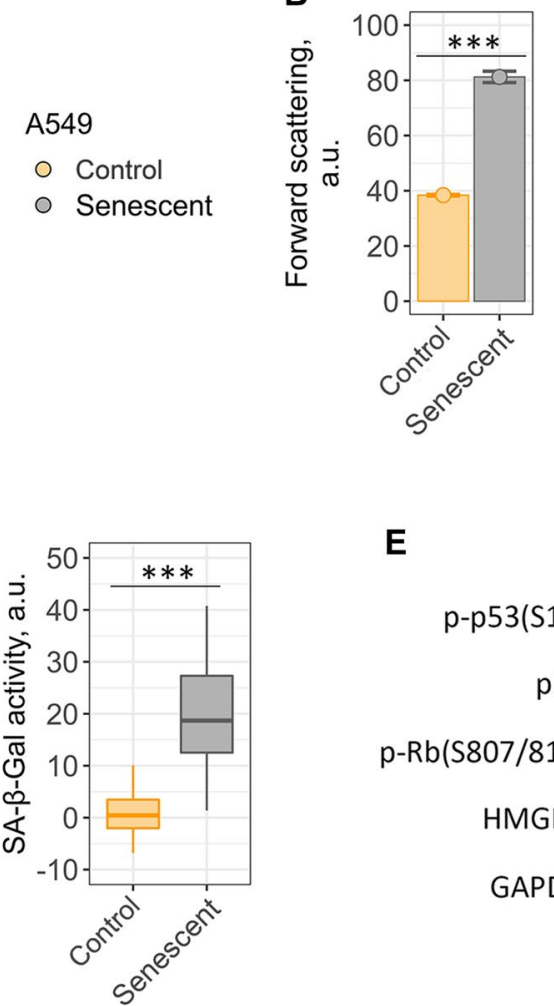

E

C
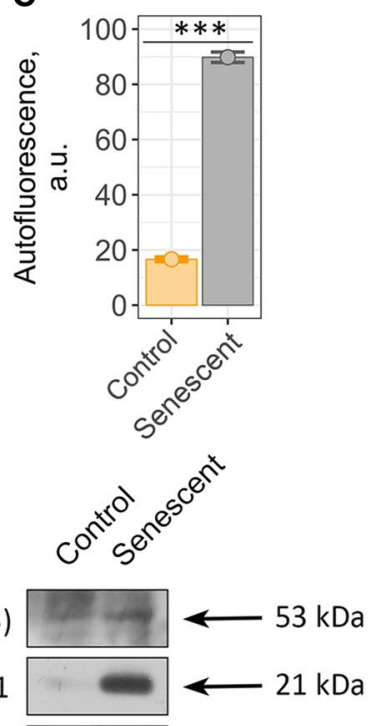

$\mathrm{p}-\mathrm{Rb}(\mathrm{S} 807 / 811)$

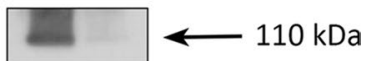

HMGB1
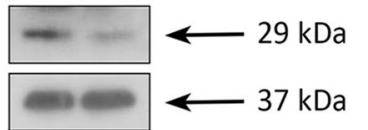

Fig. 6 Validation of etoposide-induced A549 senescence model. Senescent A549 cells display a loss of proliferation, b acquire elevated cell size, $\mathbf{c}$ autofluorescence level and d SA-b-Gal activity compared to the control ones. e Phosphorylation levels of p53 and $\mathrm{Rb}$ and expression levels of p21 and HMGB1 proteins in control and senes- cent A549. Values presented are mean \pm SD. For multiple groups comparisons at a one-way ANOVA was applied, $n=3, n s$ not significant, $* * * p 0.001$. For pair comparisons at $\mathbf{b}, \mathbf{c}$ and $\mathbf{d}$ Welch's $t$ test was used, $n=3$ for $\mathbf{b}$ and $\mathbf{c}, n=50$ for $\mathbf{d}$, $* * * p<0.001$. Scale bars for images are $500 \mu \mathrm{m}$. GAPDH was used as loading control 
A
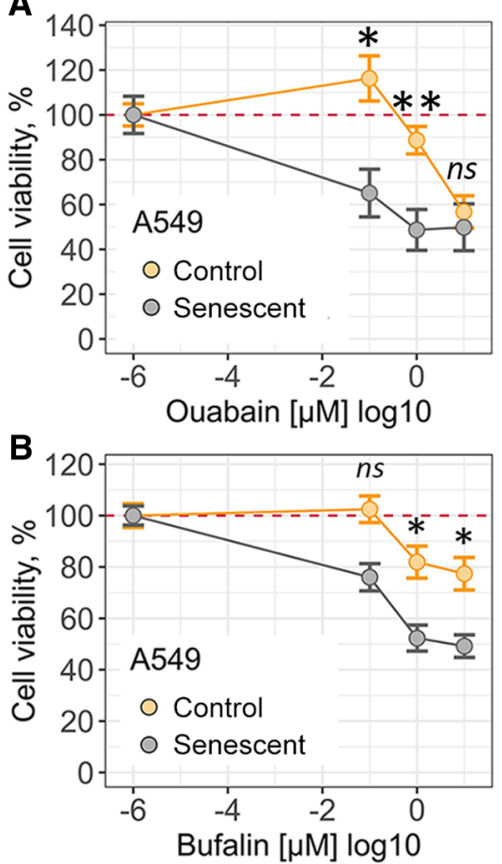

D

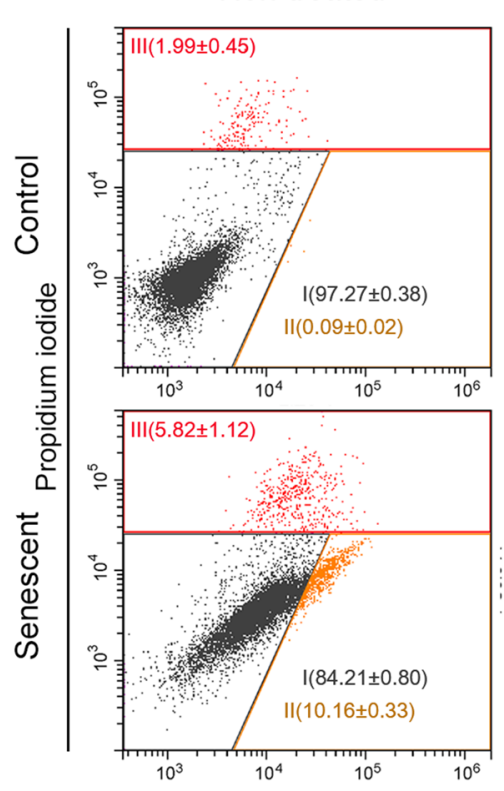

C

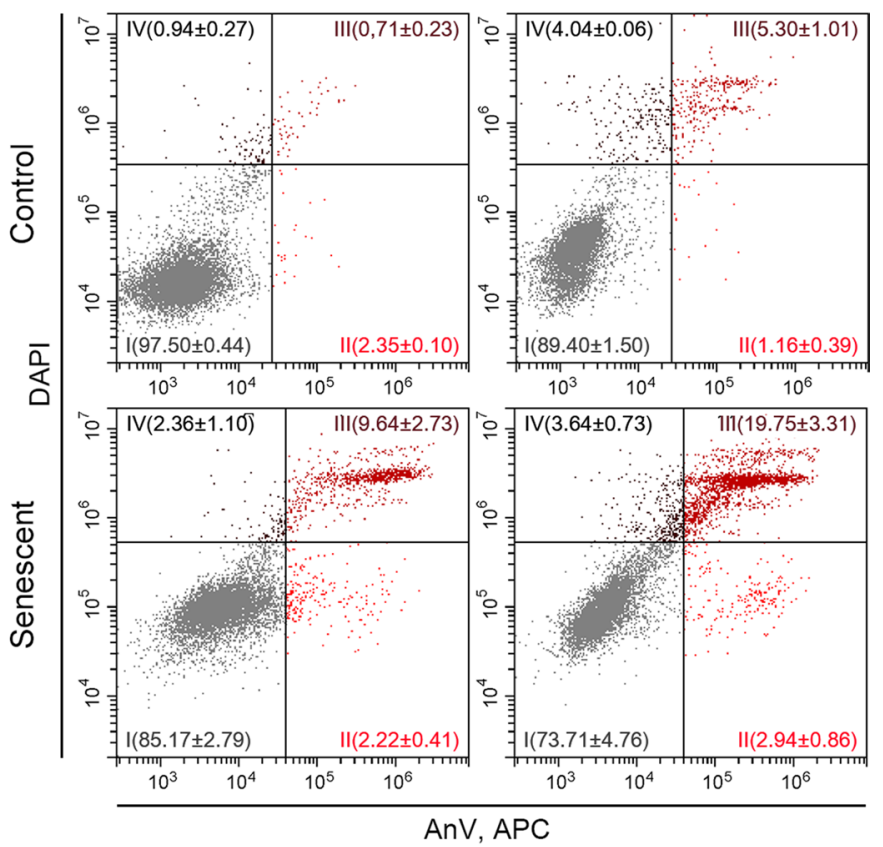

Ouabain-treated, day 1
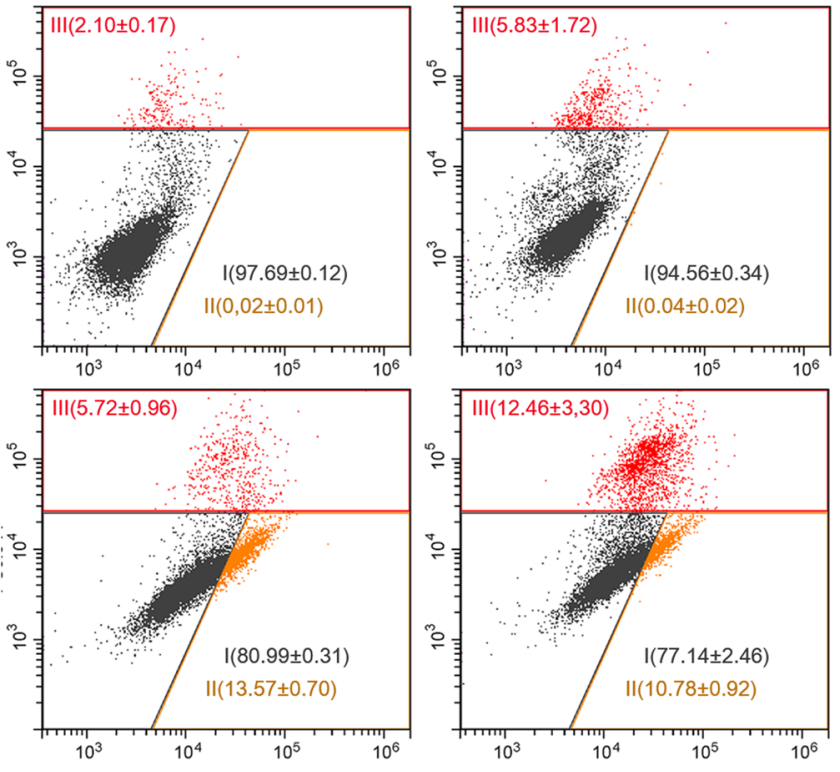

Activity of caspases 3 and 7, FITC

Fig. 7 Cardiac glycosides induce cell death in etoposide-treated senescent A549 cells. a, b Relative cell viability (\%) of control and senescent A549 in 3 days after treatment with $10^{-7}, 10^{-6}, 10^{-5} \mathrm{M}$ ouabain/bufalin. c Apoptosis induction in A549 upon $10^{-6} \mathrm{M}$ ouabain assessed by Annexin V/DAPI double staining. d Activity of Cas-

of control and senescent END-MSCs performed by us. The second one was the dataset for control and senescent A549 downloaded from GEO [43]. Of note, senescence inducing conditions coincided with those applied in the present study pase-3/7 estimated in control and senescent A549 treated with $10^{-6} \mathrm{M}$ ouabain. $n=3$ independent experiments. All data correspond to the mean \pm SD. Statistical significance was assessed by the Welch's $t$ test: $n s$ not significant, $* p<0.05, * * p<0.01$

and in the pilot studies of cardiac glycosides-induced senolysis. And the third dataset was for control and senescent IMR-90 obtained directly from the study on cardiac glycosides as senolytic compounds [25]. Importantly, IMR-90 

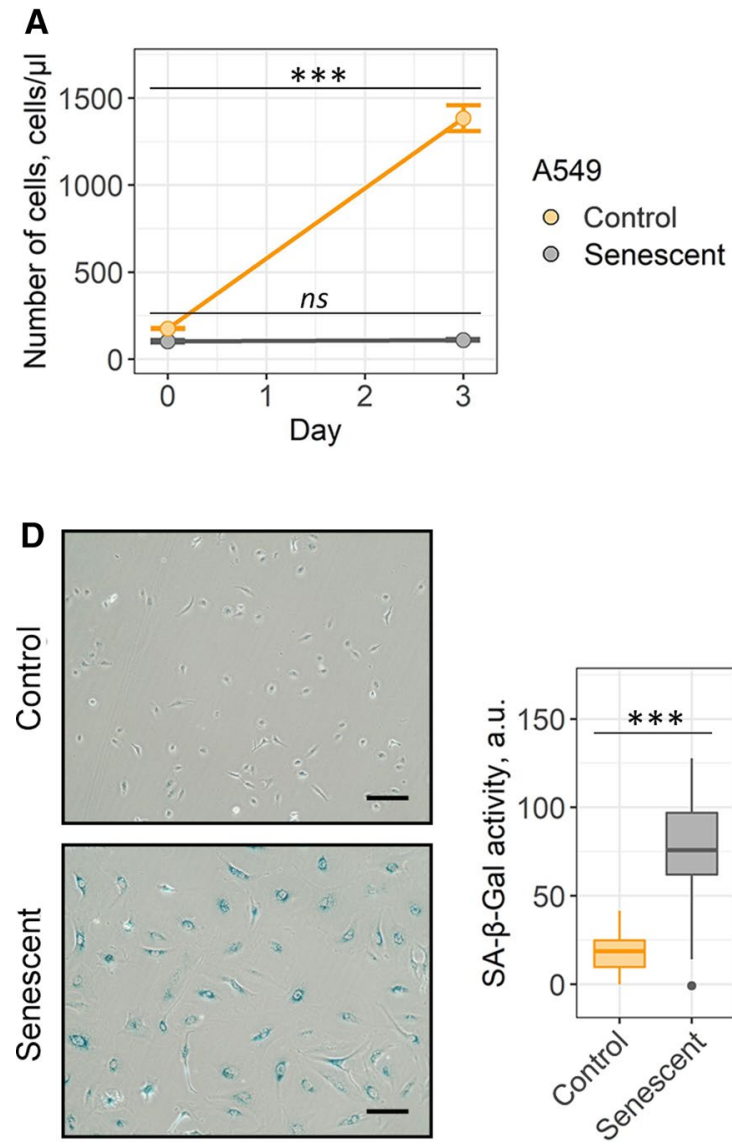

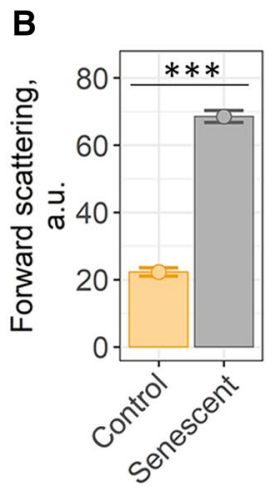

C

E

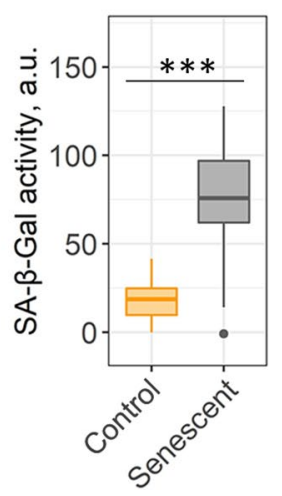

Fig. 8 Senolytic action of cardiac glycosides on doxorubicin-treated senescent A549: a proliferation ability, b cell size, $\mathbf{c}$ autofluorescence levels and d SA- $\beta$-Gal activity, e phosphorylation level of $\mathrm{Rb}$ and expression levels of p21 and HMGB1 proteins. f Relative cell viability $(\%)$ of control and senescent cells after treatment with $10^{-6} \mathrm{M}$ ouabain/bufalin, respectively. Values are mean \pm SD. For multiple groups

were proved to be prone for ouabaine-induced senolysis. It should be noted that comparing only two datasets relevant for END-MSCs and A549 would ultimately lead to improper results, as in such analysis the sought-for difference between senescence process in END-MSCs and A549 would be indistinguishable from the variations mediated by diverse technical batch effects (i.e. type of sequencing machine, the conditions of the sample ran). To minimize any batch effects, we compared one dataset for ouabain-resistant cells (ENDMSCs) and two for ouabain-sensitive cells (A549, IMR-90). To validate the relevance of the further comparative analysis, we performed a Principal Component Analysis for the subset of genes related to the GO term "cellular senescence" (GO 0090398) based on the summary dataset containing samples from all three described experiments. Indeed, for each cell type samples clustered into two separate groups- the control and the senescent ones (Fig. 10d).
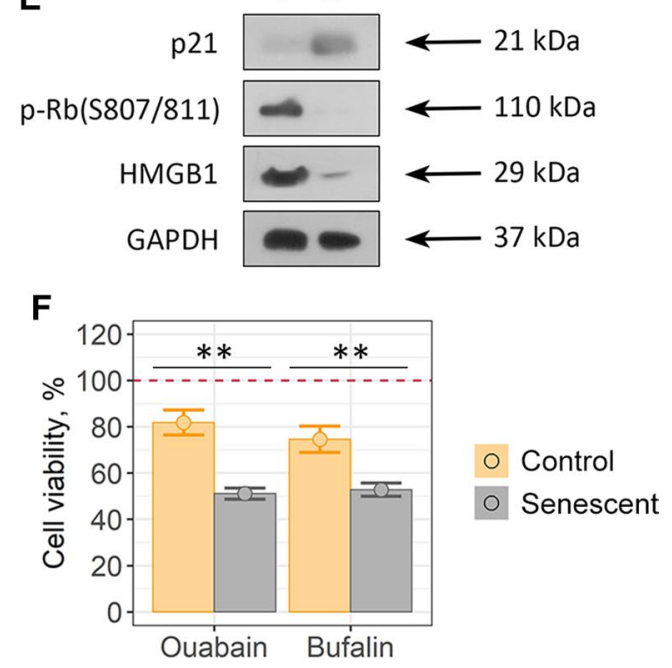

comparisons at a one-way ANOVA was applied, $n=3, n s$ not significant, $* * p<0.01, * * * p<0.001$. For pair comparisons at b, c, d and $\mathbf{f}$ Welch's $t$ test was used, $n=3$ for $\mathbf{a}, \mathbf{b}, \mathbf{c}, \mathbf{f} n=50$ for $\mathbf{d}, n s$ not significant, $* p<0.05, * * p<0.01$, ***p $<0.001$. Scale bars for images are $500 \mu \mathrm{m}$. GAPDH was used as loading control

To perform analysis of differentially expressed genes (DEGs) during senescence of ouabain-resistant and -sensitive cells, we generated the statistical model that summed up two predictors and their interaction. The schematic presentation of the analysis is displayed in Fig. 10c. In brief, the first predictor in the model divided all samples by control and senescence subgroups, the second-by ouabain resistance or ouabain sensitivity and the last component in the model reflected the interaction of both predictors. The result of differential expression testing for the last variable is presented in Supplementary Table 1.

We then conducted gene set enrichment analysis (GSEA) in Gene Onthology (GO) terms for Biological Processes (BP) (Supplementary Table 2). Notably, among the significantly enriched processes we found 'Potassium ion import' and 'Positive regulation of cation transmembrane transport' to be up-regulated during senescence 
A
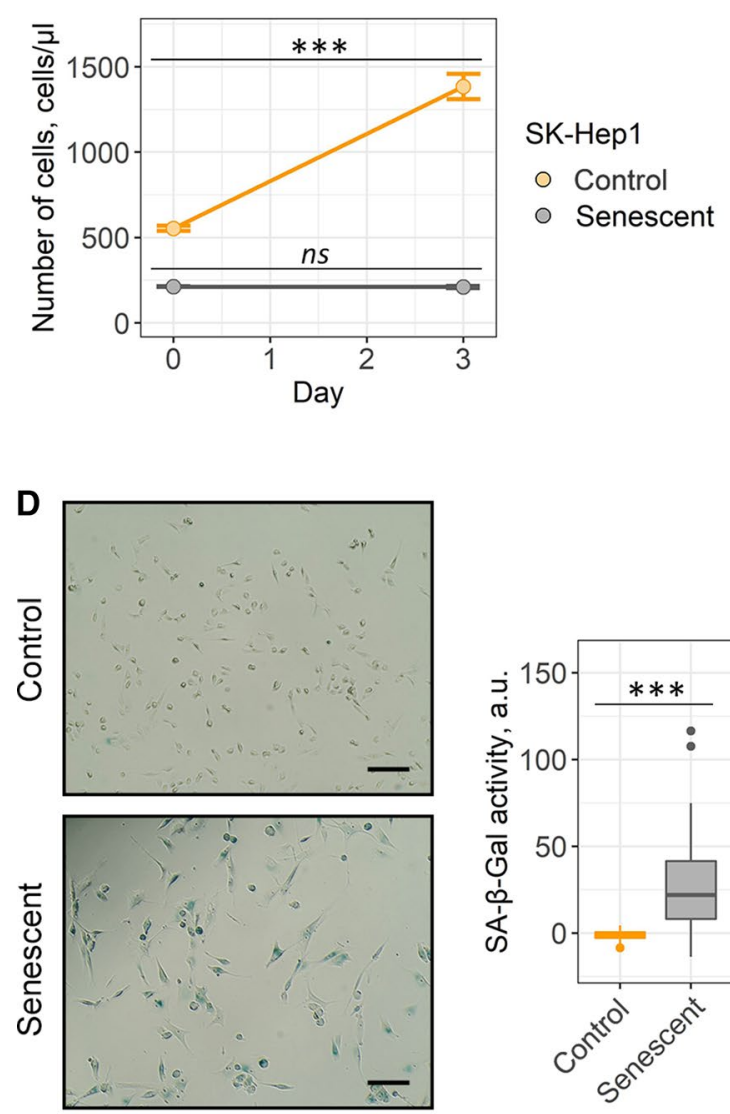

B

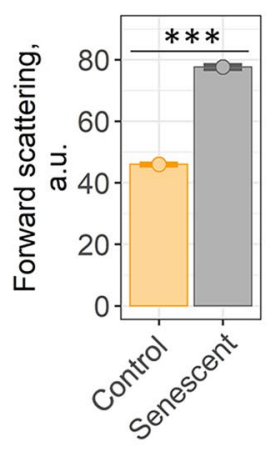

E

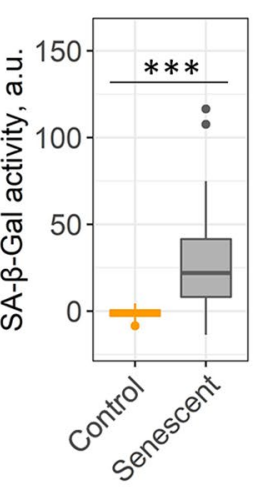

$\mathbf{F}$
Fig. 9 Ouabain/bufalin-induced senolysis in etoposide-treated senescent SK-Hep1: a proliferation ability, b cell size, $\mathbf{c}$ autofluorescence levels and d SA- $\beta$-Gal activity, e phosphorylation level of $\mathrm{Rb}$ and expression levels of $\mathrm{p} 21$ and HMGB1 proteins. f Relative cell viability (\%) of control and senescent cells after treatment with $10^{-6} \mathrm{M}$ ouabain/bufalin, respectively. Values are mean \pm SD. For multiple groups

of ouabain-resistant END-MSCs as compared to senescence of ouabain-sensitive cells (Fig. 10e). The core enrichment gene lists related to these processes included KCNJ2, KCNJ14, KCNJ8, SLC12A7, SLC12A8, WNK4 which were significantly upregulated in senescent ENDMSCs (Fig. 10f). The proteins encoded by KCNJ genes are inward-rectifier type potassium channels, which have a greater tendency to allow potassium to flow into a cell rather than out of a cell. SLC12 is a family of cation-coupled chloride transporters. WNK4 gene encodes for serine/ threonine kinase that acts as an activator of sodium-coupled chloride cotransporters and inhibitor of potassiumcoupled chloride cotransporters.

Together, these findings allowed suggesting that senescent END-MSCs can restore depleted intracellular $\mathrm{K}^{+}$levels more effectively than senescent A549 cells and thus can manage with ouabain-induced ionic disbalance.
C
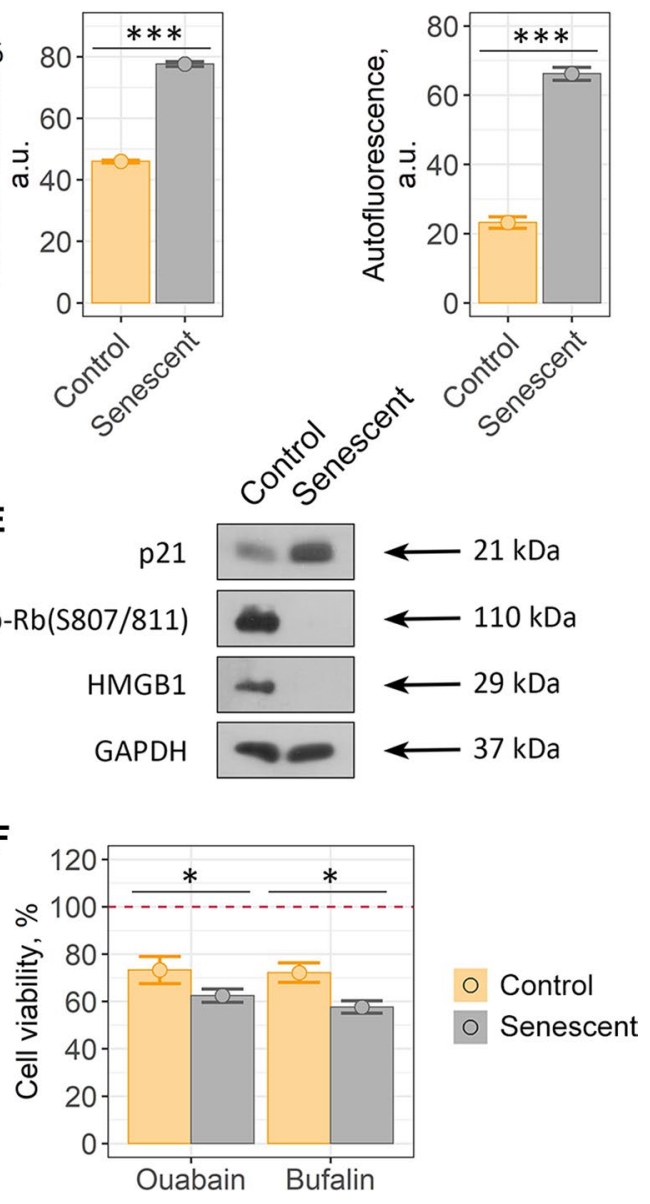

comparisons at a one-way ANOVA was applied, $n=3$, $n s$ not significant, $* * p<0.01, * * * p<0.001$. For pair comparisons at $\mathbf{b}, \mathbf{c}, \mathbf{d}$ and $\mathbf{f}$ Welch's $t$ test was used, $n=3$ for $\mathbf{a}, \mathbf{b}, \mathbf{c}, \mathbf{f} n=50$ for $\mathbf{d}, n s$ not significant, $* p<0.05, * * p<0.01, * * * p<0.001$. Scale bars for images are $500 \mu \mathrm{m}$. GAPDH was used as loading control

\section{Senescent END-MSCs display elevated apoptosis-resistance compared to the control ones, while senescent A549 do not}

The role for intracellular $\mathrm{K}+$ in the regulation of cell death is well established [46]. Revealed differences between senescent END-MSCs and A549 cells in the ability to maintain intracellular $\mathrm{K}+$ homeostasis prompted us to compare the overall stress resistance acquired during senescence of both cell types. It is known that exogenous stresses are accompanied by the disturbance in ionic homeostasis, leading to a rapid exchange of various ions, including $\mathrm{K}^{+}$between the cell and its environment [46]. The outcomes of stressful influences are largely dependent on the ability of cells to restore the appropriate intracellular ionic balance. As shown in Fig. 10e, f, senescence of END-MSCs was accompanied by the upregulation of $\mathrm{K}^{+}$import suggesting that during 

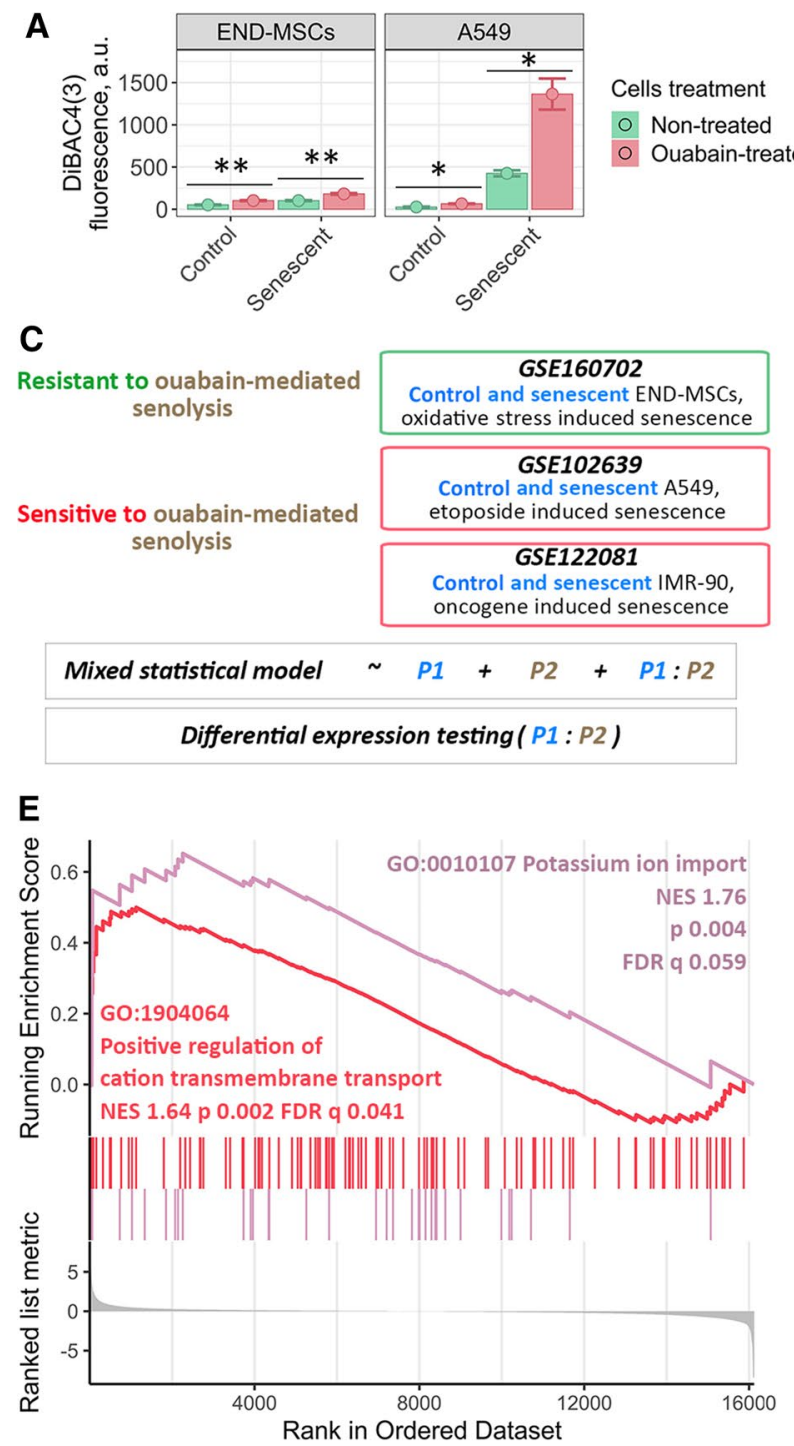

Fig. 10 Ouabain-resistant senescent END-MSCs are able to restore disturbance of $\mathrm{K}^{+} / \mathrm{Na}^{+}$homeostasis caused by ouabain via effective $\mathrm{K}^{+}$import, while ouabain-sensitive senescent cells lack this ability. a Membrane potential determination of control and senescent ENDMSCs and A549 cells before and $24 \mathrm{~h}$ after ouabain treatment, using fluorescent probe DiBAC4(3). b Mitochondrial membrane potential evaluation of control and senescent END-MSCs and A549 cells before and $24 \mathrm{~h}$ after ouabain treatment, using fluorescent probe JC-1. c Design of the comparative bioinformatic analysis of three independent RNA-Seq datasets. d Principal Component Analysis for the subset of genes related to the GO term "cellular senescence" (GO 0090398)

senescence development END-MSCs enhance their ability to restore $\mathrm{K}^{+}$levels. Interestingly, we were not able to reveal similar tendency for A549 senescence, thus, A549 seemed not to acquire any specific features related to the $\mathrm{K}^{+}$import during senescence progression. According to the literary data, stress-induced decrease in intracellular $\mathrm{K}^{+}$and inability to restore its level favor activation of caspases and nucleases and thus are proposed to be a pro-apoptotic factors [46].
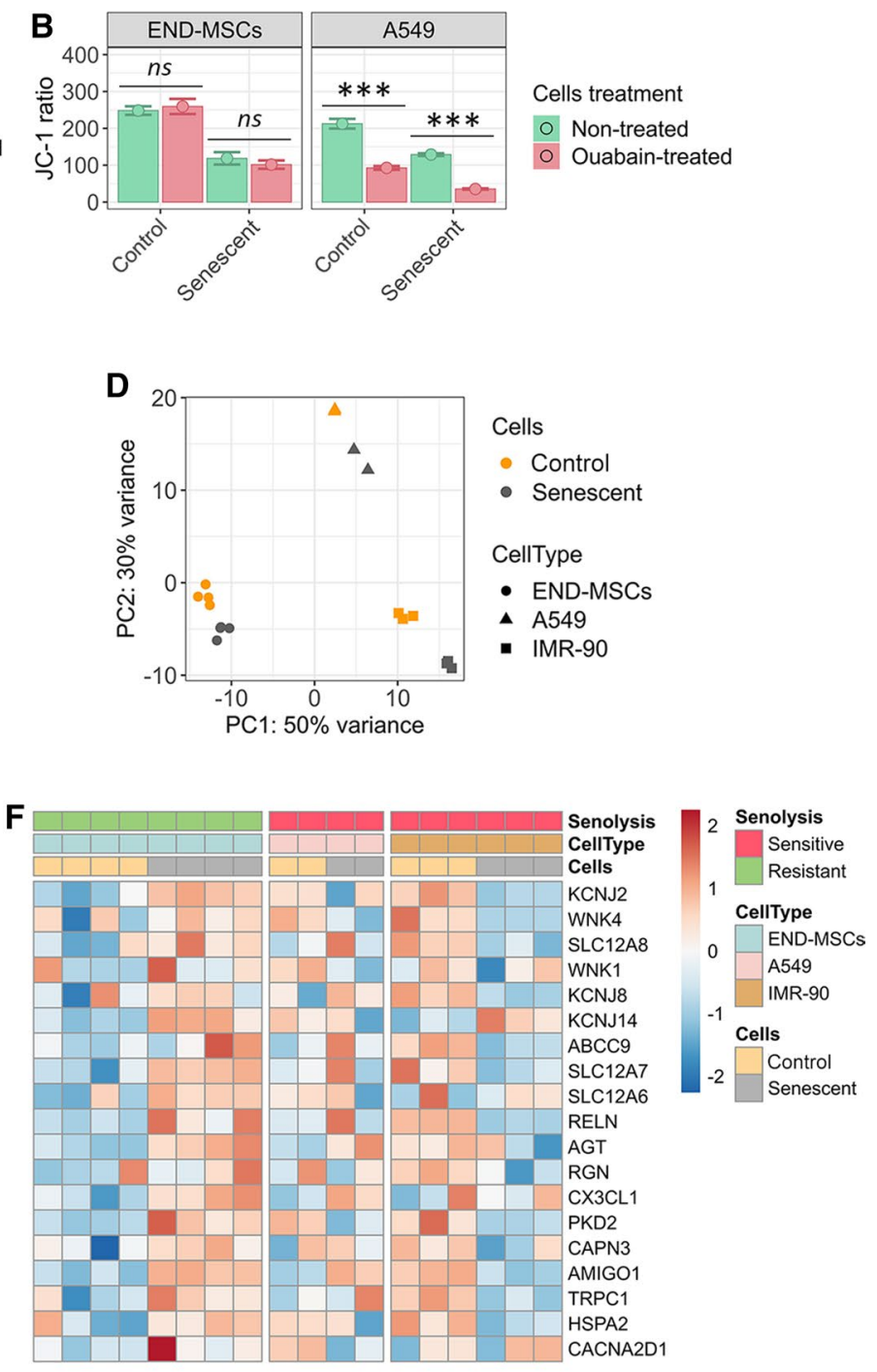

based on the combined data from three independent RNA-Seq datasets. e GSEA results for the differentially expressed genes between the cells resistant to ouabain-mediated senolysis (END-MSCs) and ones sensitive to ouabain-mediated senolysis (A549 and IMR-90) for 'Positive regulation of cation transmembrane transport' and 'Potassium ion import' biological processes. $\mathbf{f}$ Heatmap reflecting the core enrichment genes for 'Positive regulation of cation transmembrane transport' and 'Potassium ion import' biological processes. Values are mean \pm SD. Statistical significance was assessed by the Student's $t$ test: $n s$ not significant, $* p<0.05, * * p<0.01, * * * p<0.001$

In line with this suggestion, using bioinformatic analysis of the above RNA-seq datasets we revealed significant down-regulation of the processes related to apoptosis during senescence of END-MSCs as compared to A549 and IMR-90 (Fig. 11a). Moreover, senescence of A549 and IMR-90 characterized by significant up-regulation of pro-apoptotic genes, including $B A D, B A X, B O K, B A K-1$, NOXA (Fig. 11b). These data indicate that END-MSCs acquire apoptosis-resistant 
phenotype during senescence, while senescent IMR-90 and A549 became apoptosis-prone (Fig. 11b). To strengthen this observation, we additionally analyzed microarray dataset for control and replicatively senescent AD-MSCs that also preserved ouabain-resistance during senescence as indicated above (Fig. 4g). When analyzing for the expression levels of the apoptosis-related genes, we observed distinct apoptosisresistant phenotype of senescent AD-MSCs similar to that of senescent END-MSCs (Supplemental Fig. S8a, b).

To verify results of transcriptomic analysis, we estimated expression levels of genes related to apoptosis and $\mathrm{K}^{+}$import in both types of senescent cells-END-MSCs and A549 by RT-PCR (Supplemental Fig. S9). Additionally, we analyzed expression of the same gene list using other ouabain-resistant and ouabain-sensitive cellular models, doxorubicin-treated senescent DP-MSCs and etoposidetreated senescent SK-Hep1, respectively (Supplemental Fig. S9). As shown in Supplemental Fig. S9, both types of ouabain-resistant cells - senescent END-MSCs and DPMSCs displayed similar gene expression pattern, specifically $K C N J 2, S L C 12 A$ and $W N K 4$ participating in $\mathrm{K}+$ restoration were upregulated, proapoptotic $B A X$ was downregulated, while antiapoptotic $M C L-1$ was upregulated. Moreover, the expression pattern of the same genes differed from those observed for ouabain-sensitive A549 and SK-Hep1. These results confirm our transcriptomic data and reinforce the conclusion regarding varied apoptosis profile acquired during senescence of different cell types.

We next tested whether the observed distinctions in apoptosis background during senescence of END-MSCs and A549 may have an impact on the overall stress resistance of senescent cells. To do so, we assessed cell viability upon oxidative stress and staurosporine, typically applied to induce apoptosis. Indeed, senescent END-MSCs turned out to be significantly more resistant towards both stresses compared to their control counterparts (Fig. 11c). Contrarily, A549 cells demonstrated vice versa situation, as senescent cells displayed tendency to be more sensitive towards stressinduced cell death (Fig. 11d). This observation shifts the existing paradigm that enhanced death resistance is a common feature of any kind of senescent cells, demonstrating its obvious cell specificity.

Based on the above data, we assumed that reducing apoptosis "defense" of senescent END-MSCs should create favorable conditions for further senolysis. In this regard, we focused on a well-known antiapoptotic member of BCL-2 family proteins-MCL-1, since its expression was upregulated in apoptosis-resistant senescent END-MSCs (Fig. 11b and Supplemental Fig. S9a). To this end, we pretreated control and senescent END-MSCs with A-1210477 the specific MCL-1 inhibitor and then applied cardiac glycosides to induce senolysis. Of note, A-1210477 itself had no effect on the proliferation capacity of control cells and on the viability of senescent ones as indicated on the growth curves (Fig. 11e). Subsequent application of cardiac glycosides led to the decrease in a number of viable cells, however the effect was more pronounced in senescent ESCs (Fig. 11f). Therefore, inhibiting MCL-1 with A-1210477 predisposed senescent ESCs towards ouabain-induced death indicating for senolysis. The latter evidence for apoptosisresistance acquired during END-MSCs senescence to be an intrinsic barrier for effective senolysis triggered by cardiac glycosides.

\section{Discussion}

Within the present study we tested senolytic effects of cardiac glycosides, namely ouabain and bufalin, towards hMSCs. Both compounds were recently identified to have selective cytotoxic effect on senescent cells (oncogene-/therapy-induced senescence models) of different origins, including primary cells IMR-90, HUVEC, ARPE-19, T/C-28 and cancer cells SK-Hep1, A549, SK-Mel-5, MCF7, HCT116, HaCat, H1299, U373-MG, H1755 [25, 26]. Unexpectedly, we were not able to reveal any preferential cytotoxic effects neither of ouabain nor of bufalin on senescent END-MSCs compared to the control ones. Importantly, the absence of senolysis was verified using hMSCs obtained from adipose tissue, dental pulp and Warton jelly and besides various senescence models. The latter led us to the suggestion that resistance to cardiac glycoside-induced senolysis might be the common feature for hMSCs. At the same time we were able to induce apoptosis preferentially in senescent A549 and SK-Hep1, thus reproducing senolytic effect of cardiac glycosides described in the relevant articles [25, 26]. With cells resistant and sensitive to ouabain-induced senolysis (ouabain-resistant/ouabain-sensitive) available, we tried to elucidate fundamental causes underlying this difference.

The common molecular mechanism of cardiac glycosides' action is binding to the $\mathrm{Na}^{+} / \mathrm{K}^{+}$-ATPase and blocking its activity. By hydrolyzing ATP this enzyme ensures pumping $\mathrm{Na}^{+}$out of the cell and importing $\mathrm{K}^{+}$into the cells and thus maintains physiological electrochemical gradient, ionic homeostasis, cellular $\mathrm{pH}$ and cell volume that are essential for cell survival and functioning [47]. Therefore, we speculated that the senolytic ability of ouabain might depend on the severity of $\mathrm{K}^{+} / \mathrm{Na}^{+}$disbalance in the treated cells. In line with this suggestion, we revealed that ouabain induced more pronounced depolarization of plasma membrane and drop of MMP in ouabain-sensitive senescent A549.

Generally speaking, the impact of $\mathrm{Na}^{+} / \mathrm{K}^{+}$-ATPase inhibiting on cell survival was shown to be cell-type specific. For example, ouabain was shown to potentiate apoptosis in lymphocytes, Jurkat cells, canine epithelial cells, while it failed to induce death in epithelial cells from rat aorta, rat 


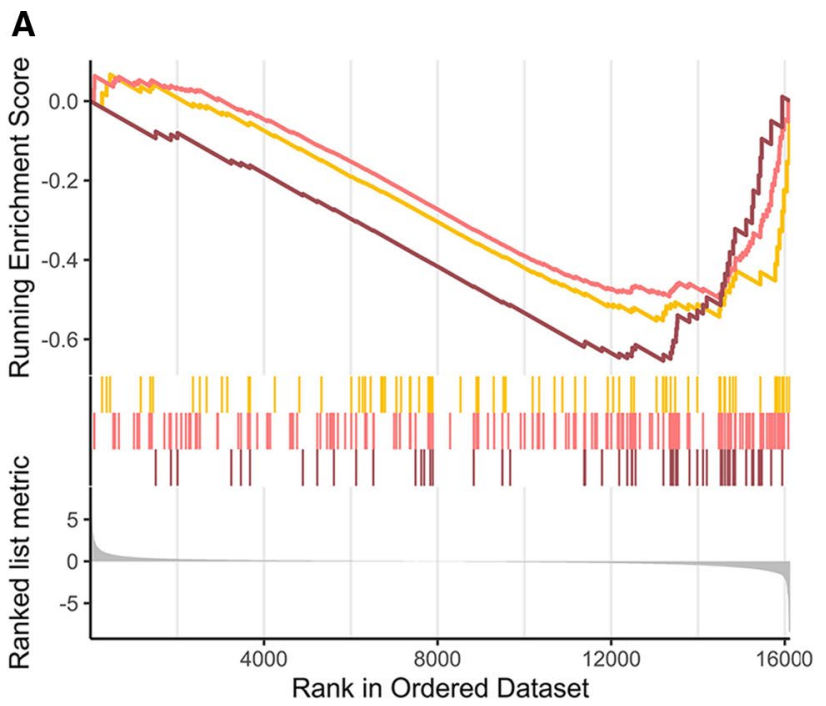

B

GO:0006919 Activation of cysteine-type endopeptidase activity involved in apoptotic process NES $-1.54 p 0.009$ FDR q 0.101

GO:2001235 Positive regulation of apoptotic signaling pathway NES -1.51 p 0.003 FDR $q 0.052$

GO:1902110 Positive regulation of mitochondrial membrane permeability involved in apoptotic process NES $-1.74 p<0.001$ FDR $q 0.020$

C

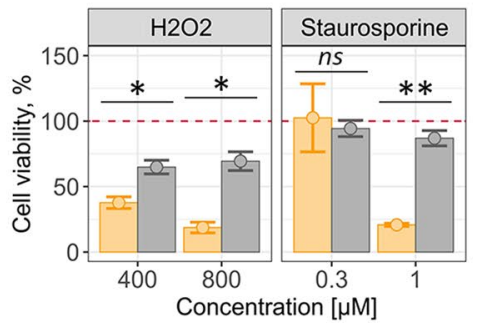

END-MSCs

O Control

O Senescent

\section{E}

A549

- Control

O Senescent

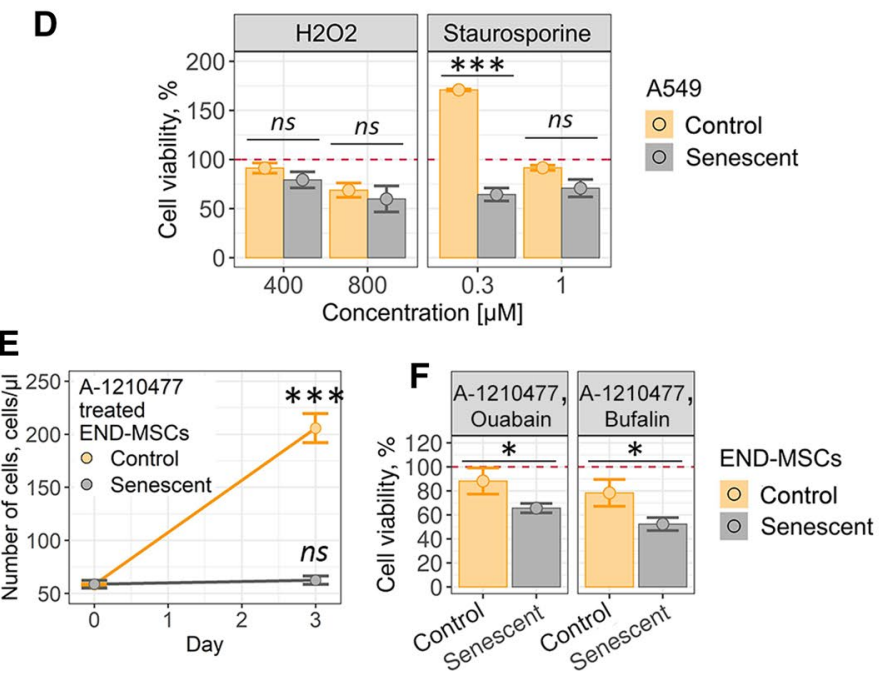

END-MSCs

O Control

O Senescent
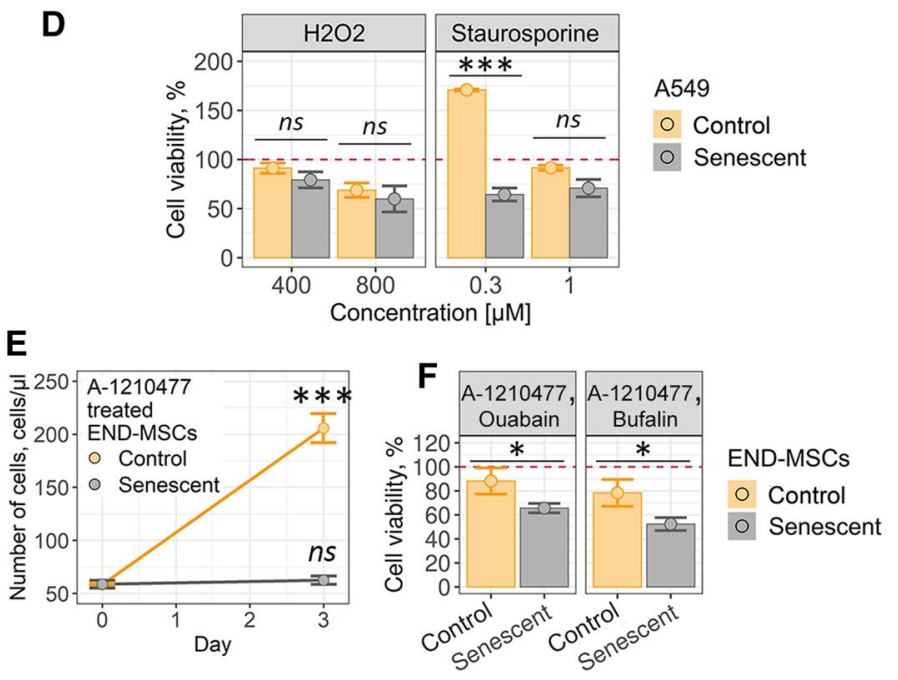

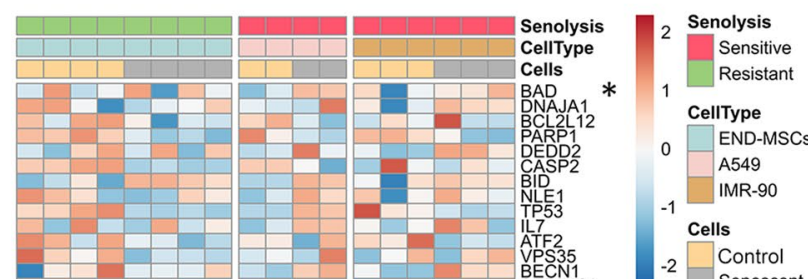

$-2 \square \begin{aligned} & \text { Control } \\ & \text { Senescent }\end{aligned}$
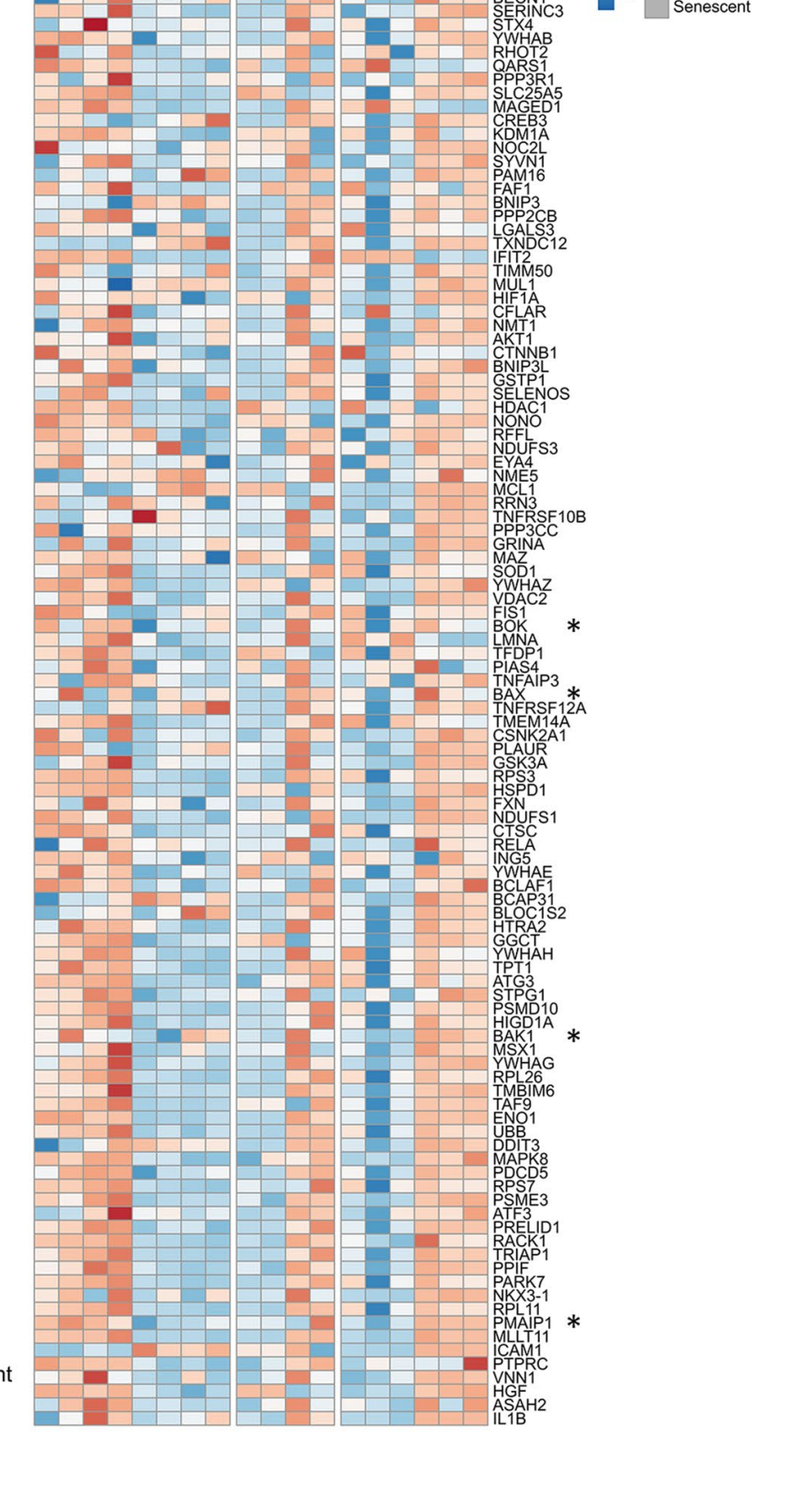

cerebral granule cells and porcine renal proximal tubular LLC-PK1 lymphocytes despite similar modulation of the cationic ratio [48-54]. One of the proposed mechanisms of proapoptotic ouabain action is depletion of intracellular $\mathrm{K}^{+}$ that favors apoptotic shrinkage, activation of caspases and initiation of apoptotic programming [46, 47]. Considering

comparable $\mathrm{K}^{+}$loss but opposite effect on death induction obtained for ouabain-resistant and ouabain-sensitive models, we suggested the differences in cation's compensatory systems. The drop in intracellular $\mathrm{K}^{+}$level should essentially lead to the activation of the $\mathrm{K}^{+}$import from the extracellular space to compensate the lack of this cation. Therefore, 
४Fig. 11 Senescence of ouabain-resistant END-MSCs is accompanied by acquisition of apoptosis-resistant phenotype, while ouabainsensitive cells become apoptosis-prone during senescence. a GSEA results for the DEGs between the cells resistant to ouabain-mediated senolysis (END-MSCs) and the ones sensitive to ouabain-mediated senolysis (A549 and IMR-90) in the apoptosis-related terms. b Heatmap reflecting expression of the core enrichment genes for apoptosisrelated terms from a. The common pro-apoptotic genes are marked with asterisks. c, d Relative cell viability (\%) of control and senescent END-MSCs or A549 treated either with $\mathrm{H}_{2} \mathrm{O}_{2}$ or staurosporine at indicated concentrations. e Growth curves of control and senescent END-MSCs treated with A-1210477. f Relative cell viability (\%) of control and senescent END-MSCs pretreated with A-1210477 followed by ouabain or bufalin supplementation. Red line marks the initial percent of viable cells (prior to treatment) normalized to $100 \%$ both for the control and senescent ESCs. Values are mean \pm SD. Statistical significance was assessed by the two-tailed Student's $t$ test: ${ }^{*} p<0.05,{ }^{* *} p<0.01, * * * p<0.001, n s$ not significant

the effectiveness of $\mathrm{K}^{+}$restoration might underlie apoptosis predisposition upon ouabain treatment. To test this suggestion, we applied complex bioinformatic analysis comparing alterations in transcriptomic signatures during senescence of ouabain-resistant cells (END-MSCs) and ouabain-sensitive cells (A549 and IMR-90). We observed strong upregulation of the processes related to potassium ion import during END-MSCs senescence, while during senescence of ouabain-sensitive A549 and IMR-90 these processes remained unchanged or even decreased. Based on that we can speculate that senescent END-MSCs can effectively cope with ouabain-induced $\mathrm{K}^{+}$depletion via active cation importing systems, what prevents apoptosis induction. Contrarily, ouabain-sensitive senescent A549 and IMR-90 seem to be less able to overcome $\mathrm{K}^{+}$loss, thus are apoptosis-prone. In favor of this assumption, ouabain induced more significant plasma and mitochondrial membranes depolarization in senescent A549 cells, demonstrating pronounced ionic disbalance typical for dying cells.

Rather than being the unique feature of ouabain-induced apoptosis, drop of intracellular $\mathrm{K}^{+}$content is probably the common characteristic of apoptotic death triggered by various stresses, e.g. staurosporine treatment and oxidative stress [46]. Accordingly, decreased ability of senescent A549 to restore cytoplasmic $\mathrm{K}^{+}$should ultimately lead to decline in the overall stress resistance. However, this notion contradicts with the modern definition of cell senescence, which states that senescent cells are highly resistant to apoptosis [16]. It should be specifically highlighted that enhanced apoptosis resistance formed the basis for senolytics development [16, 17].

The first mention of the increased stress resistance of senescent cells dates back to 1995 , when replicatively senescent fibroblasts were found to be more resistant to serum withdrawal compared to the control ones [55]. This initial study was followed by the limited number of investigations indicating that senescent cells are more resistant to UV-light, staurosporine, thapsigargin and other stresses than their proliferating counterparts $[56,57]$. However, we are far from the first to raise the question of the enhanced apoptosis-resistance as the general feature of senescent cells. Thus, in the review published in 2003 it was reported “... apoptosis resistance is not a general feature of senescent cells, which may also be apoptotic prone depending on cell type and apoptotic stimuli..." [58]. Indeed, several data demonstrated higher sensitivity of senescent cells to stress-induced apoptosis than of control cells [59-61]. For example, senescent HUVECs were more prone to apoptosis induced by oxidized LDL or TNF $\alpha$ compared to control cells [61]. Despite the obvious controversy, the last doubt appeared in 2015 and sounded as follows: "It seems doubtful that global apoptosis resistance is a general feature of senescence cells" [62]. In the same year, the first study uncovering senolysis was published [16]. Within this study, the authors highlighted increased expression of prosurvival gene networks in senescent cells that contributed to their enhanced resistance to apoptosis. Thus, initially senolytics represented the drugs to target proteins that protected senescent cells from apoptosis. The discovery of senolytics together with the impressive results of senescent cells' removal using INK-ATTAC transgenic mice triggered a flurry of studies searching for the new compounds with senolytic activity [16-26, 63]. Over the last two years a plenty of reviews on senolytics was published [13-15, 64-66]. However, since 2015 enhanced apoptosis resistance of senescent cells was not the matter of debate, though, in fact, it was not tested within these studies.

Interestingly, when comparing transcriptomic signatures of ouabain-resistant cells (END-MSCs) and ouabain-sensitive cells (A549 and IMR-90), we found that only senescence of END-MSCs was accompanied by acquisition of noticeable anti-apoptotic profile. On the contrary, senescence of A549 and IMR-90 characterized by significant up-regulation of pro-apoptotic genes, including $B A D, B A X, B O K, B A K-1$, $N O X A$ and so on. Importantly, these results were extended using other ouabatin-resistant cells DP-MSCs. On the one hand, these results provide additional molecular explanation for the absence of ouabain-induced senolysis in hMSCs, and, on the other hand, clearly demonstrate that A459 and IMR90 become predisposed to apoptosis during senescence. Of note, our data regarding transcriptomic alterations in genes related to apoptosis in senescent IMR-90 coincided with the results presented, yet not discussed, in the article published by Guerrero et al., as the datasets for control and senescent IMR-90 cells originated from this study [25]. The precise analysis of the heatmap provided within this study revealed upregulation of $B A X, B I D, B A D, B A K 1$ and $N O X A$ in senescent IMR-90 as compared to the control cells. Furthermore, Baar et al. also revealed "unexpected" upregulation of proapoptotic and downregulation of antiapoptotic genes 
in senescent IMR-90, mentioning that senescent IMR-90 should be primed to undergo apoptosis [21].

In order to strengthen our observation, we compared resistance of control and senescent END-MSCs and A459 to the most common apoptosis-inducing stimuli-oxidative stress and staurosporine. According to the results of our bioinformatic analysis, senescent END-MSCs turned out to be far more stress-resistant than control cells. At the same time, senescent A549 demonstrated the opposite reaction being slightly more susceptible than control cells to both types of stressful stimuli. Therefore, senolytic action of cardiac glycosides, at least for A549, might be mediated by the predisposition of senescent cells of this origin to apoptosis compared to their control counterparts, whereas the absence of ouabain-induced senolysis in hMSCs might be explained by the increase in overall apoptotic resistance during senescence of these cells. Moreover, weakening "antiapoptotic defense" by MCL-1 inhibition predisposed END-MSCs to senolysis induced by cardiac glycosides. Although we were able to achieve the desired senolysis of END-MSCs by inhibiting MCL-1, the strategy to modulate expression of the concrete anti-apoptotic genes to predispose senescent hMSCs to senolysis does not seem very promising, since the expression dynamics of the concrete anti- or pro-apoptotic genes might differ between cells of various origins. We believe that acquisition of so-called "anti-apoptotic" profile during cell senescence, rather than alterations in the concrete anti- or pro-apoptotic genes, is responsible for ouabain resistance, in this regard the possibility to switch the whole profile might seem more reasonable.

Our conclusions highlighting apoptosis-resistance acquired during hMSCs senescence to be an intrinsic barrier for effective senolysis might be extended far beyond cardiac glycosides. Notably, senescent hMSCs were found to be resistant towards other senolytic compounds. Indeed, by analyzing the existing evidence we found that various compounds with the stated senolytic activity, including navitoclax, nicotinamide riboside, danazol geldanamycin, ganetespib, fisetin, BCL-XL inhibitors, quercetin, etomoxir, antimycin A, FOXO4-DRI, 17-DMAG turned to be ineffective for the targeted removal of senescent human preadipocytes (fibroblast like precursor cells derived from human adipose tissue) and hMSCs from bone marrow [17-19, 36, 40].

The data regarding the absence of senolysis in hMSCs somewhat contradict with the inspiring results obtained using in vivo mice models, demonstrating positive outcomes upon systemic application of senolytics [37, 38]. For example, it was shown that clearance of senescent salivary gland stem cells using ABT263 may prevent radiotherapy-induced xerostomia [38]. Furthermore, removal of senescent bone marrow MSCs by quercetin improved bone marrow formation [37]. Importantly, it was shown that senescent mouse and rat MSCs unlike hMSCs are responsive to senolytics. For example, quercetin, quercetin + dasatinib, ABT263 significantly removed senescent mouse MSCs [16]. Also, 17-DMAG has been shown to greatly reduce senescent bmMSCs in a progeroid mouse model [19]. Therefore, the improvements in various organs and systems functioning described within these studies are speculated to be the consequence of the targeted removal of senescent mouse stem cells. However, these senolytics have not yet shown any functional rejuvenation of hMSCs. Such an obvious distinction between the responsiveness of mice and human MSCs towards senolysis suggests that improvements from senolytic therapies may not be conserved across the species. The results obtained for senolytic compound UBX0101 can be considered as a good illustration confirming the last statement. This agent has been shown to clear senescent cells in a mouse model of osteoarthritis, which significantly reduced pain and promoted repair of the damaged cartilage [20]. Unfortunately, UBX0101 failed to attenuate disease progression and pain in patients with moderate-to-serve painful osteoarthritis during phase II clinical trial [67-69]. The similar concerns are raised about senolytic combination dasatinib + quercetin [68].

To sum up, two important conclusions flow out from the obtained data. The former demonstrated that cardiac glycosides are unable to clear senescent hMSC. The absence of senolysis might be mediated by effective $\mathrm{K}^{+}$cellular import and increased apoptosis resistance in senescent hMSCs. The latter is more fundamental and reveals that apoptosis resistance is not a general feature of senescent cells, as during senescence some cells acquire 'apoptosisresistant' phenotype, while others do not. Importantly, only apoptosis-prone senescent A549 cells could be effectively cleared by cardiac glycosides. Based on that we can speculate that the effectiveness of other senolytic approaches might depend on whether senescent cells are indeed apoptosis-resistant compared to their proliferating counterparts. Finally, though 'senolytics' is a hot topic and a lot of inspiring data are published, conclusions regarding the effectiveness of senolysis should be taken with caution as heterogeneity of cell senescence still remains a 'puzzle'.

Supplementary Information The online version contains supplementary material available at https://doi.org/10.1007/s00018-021-03980-x.

Acknowledgements The authors are thankful to Maria Sirotkina for the assistance in the figures design.

Author contributions Supervision, conceptualization, original draft preparation, writing, formal analysis and investigation, funding acquisition [AVB]; Conceptualization, formal analysis and investigation, methodology: [PID]; Formal analysis and investigation: [ANS]. 
Funding This study was funded by the Russian Science Foundation (\#19-74-10038).

Availability of data and material All data generated or analysed during this study are included in the manuscript and supporting files. Sequencing data have been deposited in GEO under accession codes GSE160702.

Code availability The custom code used in the current study is available from the corresponding author upon request.

\section{Declarations}

Conflict of interest The authors declare no competing interests.

Ethics approval (include appropriate approvals or waivers) Not applicable.

Consent to participate (include appropriate statements) Not applicable.

Consent for publication (include appropriate statements) Not applicable.

Open Access This article is licensed under a Creative Commons Attribution 4.0 International License, which permits use, sharing, adaptation, distribution and reproduction in any medium or format, as long as you give appropriate credit to the original author(s) and the source, provide a link to the Creative Commons licence, and indicate if changes were made. The images or other third party material in this article are included in the article's Creative Commons licence, unless indicated otherwise in a credit line to the material. If material is not included in the article's Creative Commons licence and your intended use is not permitted by statutory regulation or exceeds the permitted use, you will need to obtain permission directly from the copyright holder. To view a copy of this licence, visit http://creativecommons.org/licenses/by/4.0/.

\section{References}

1. von Zglinicki $\mathrm{T}$ et al (2021) Senescence in post-mitotic cells: a driver of aging? Antioxid Redox Signal 34(4):308-323

2. Sapieha P, Mallette F (2018) Cellular senescence in postmitotic cells: beyond growth arrest. Trends Cell Biol 28(8):595-607

3. Lee S, Schmitt CA (2019) The dynamic nature of senescence in cancer. Nat Cell Biol 21(1):94-101

4. Campisi J (1996) Replicative senescence: an old lives' tale? Cell 84(4):497-500

5. Serrano M et al (1997) Oncogenic ras provokes premature cell senescence associated with accumulation of p53 and p16INK4a. Cell 88(5):593-602

6. Toussaint $\mathrm{O}$ et al (2000) Cellular and molecular mechanisms of stress-induced premature senescence (SIPS) of human diploid fibroblasts and melanocytes. Exp Gerontol 35(8):927-945

7. Roninson IB et al (2001) If not apoptosis, then what? Treatmentinduced senescence and mitotic catastrophe in tumor cells. Drug Resist Updates 4(5):303-313

8. Hernandez-Segura A et al (2018) Hallmarks of cellular senescence. Trends Cell Biol 28(6):436-453

9. Wang B et al (2020) Senescent cells in cancer therapy: friends or foes? Trends Cancer 6(10):838-857
10. Nacarelli et al (2017) Epigenetic basis of cellular senescence and its implications in aging. Genes (Basel) 8(12):343

11. Munoz-Espin et al (2014) Cellular senescence: from physiology to pathology. Nat Rev Mol Cell Biol 15(7):482-496

12. Ovadya $Y$ et al (2018) Impaired immune surveillance accelerates accumulation of senescent cells and aging. Nat Commun 9:5435

13. Kirkland JL, Tchkonia T (2020) Senolytic drugs: from discovery to translation. J Intern Med 288(5):518-536

14. Pignolo RJ et al (2020) Reducing senescent cell burden in aging and disease. Trends Mol Med 26(7):630-638

15. Robbins PD et al (2020) Senolytic drugs: reducing senescent cell viability to extend health span. Annu Rev Pharmacol Toxicol 61:779-803

16. Zhu Y et al (2015) The Achilles' heel of senescent cells: from transcriptome to senolytic drugs. Aging Cell 14(4):644-658

17. Zhu Y et al (2016) Identification of a novel senolytic agent, navitoclax, targeting the Bcl-2 family of anti-apoptotic factors. Aging Cell 15(3):428-435

18. Zhu Y et al (2017) New agents that target senescent cells: the flavone, fisetin, and the BCL-XL inhibitors, A1331852 and A1155463. Aging 9(3):955-963

19. Fuhrmann-Stroissnigg $\mathrm{H}$ et al (2017) Identification of HSP90 inhibitors as a novel class of senolytics. Nat Commun 8(1):422

20. Jeon $\mathrm{OH}$ et al (2017) Local clearance of senescent cells attenuates the development of post-traumatic osteoarthritis and creates a pro-regenerative environment. Nat Med 23(6):775-781

21. Baar MP et al (2017) Targeted apoptosis of senescent cells restores tissue homeostasis in response to chemotoxicity and aging. Cell 169(1):132-147

22. Wakita M et al (2020) A BET family protein degrader provokes senolysis by targeting NHEJ and autophagy in senescent cells. Nat Commun 11(1): 1935

23. Amor $\mathrm{C}$ et al (2020) Senolytic CAR T cells reverse senescenceassociated pathologies. Nature 583(7814):127-132

24. González-Gualda E et al (2020) Galacto-conjugation of Navitoclax as an efficient strategy to increase senolytic specificity and reduce platelet toxicity. Aging Cell 19(4):e13142

25. Guerrero A et al (2019) Cardiac glycosides are broad-spectrum senolytics. Nat Metab 1(11):1074-1088

26. Triana-Martínez F et al (2019) Identification and characterization of cardiac glycosides as senolytic compounds. Nat Commun 10(1):4731

27. da Silva ML et al (2006) Mesenchymal stem cells reside in virtually all post-natal organs and tissues. J Cell Sci $119(\mathrm{Pt}$ 11):2204-2213

28. Zhou W, Xu Y (2020) Application of mesenchymal stem cells in human diseases in mesenchymal stem cells in human health and diseases. In: El-Hashash AHK (ed) Mesenchymal stem cells in human health and diseases. Academic Press, pp 5-15

29. Zhou X et al (2020) Mesenchymal stem cell senescence and rejuvenation: current status and challenges. Front Cell Dev Biol $8: 364$

30. Burova E et al (2013) Sublethal oxidative stress induces the premature senescence of human mesenchymal stem cells derived from endometrium. Oxid Med Cell Longev 2013:474931

31. Liu J et al (2020) Senescence in mesenchymal stem cells: functional alterations, molecular mechanisms, and rejuvenation strategies. Front Cell Dev Biol 8:258

32. Griukova A et al (2019) Molecular basis of senescence transmitting in the population of human endometrial stromal cells. Aging 11(21):9912-9931

33. Turinetto V, Vitale E, Giachino C et al (2016) Senescence in human mesenchymal stem cells: functional changes and implications in stem cell-based therapy. Int J Mol Sci 17(7):1164 
34. Spehar K et al (2020) Restoring aged stem cell functionality: current progress and future directions. Stem Cells 38(9):1060-1077

35. Lee BC, Yu KR (2020) Impact of mesenchymal stem cell senescence on inflammaging. BMB Rep 53(2):65-73

36. Grezella $\mathrm{C}$ et al (2018) Effects of senolytic drugs on human mesenchymal stromal cells. Stem Cell Res Ther 9(1):108

37. Zhang D et al (2020) Senolytic controls bone marrow mesenchymal stem cells fate improving bone formation. Am J Transl Res 12:3078-3088

38. Peng X et al (2020) Cellular senescence contributes to radiationinduced hyposalivation by affecting the stem/progenitor cell niche. Cell Death Dis 11(10):854

39. Sharma AK et al (2020) The senolytic drug Navitoclax (ABT-263) causes trabecular bone loss and impaired osteoprogenitor function in aged mice. Front Cell Dev Biol 8:354

40. Rothmiller S et al (2021) Chronic senescent human mesenchymal stem cells as possible contributor to the wound healing disorder after exposure to the alkylating agent sulfur mustard. Arch Toxicol 95(2):727-747

41. Deryabin P et al (2019) Optimization of lentiviral transduction parameters and its application for CRISPR-based secretome modification of human endometrial mesenchymal stem cells. Cell Cycle 18(6-7):742-758

42. Borodkina A et al (2014) Interaction between ROS dependent DNA damage, mitochondria and p38 MAPK underlies senescence of human adult stem cells. Aging 6(6):481-495

43. Wang L et al (2017) High-throughput functional genetic and compound screens identify targets for senescence induction in cancer. Cell Rep 21(3):773-783

44. Skou JC (1988) The Na, K-pump. Methods Enzymol 156:1-2

45. Passos JF et al (2010) Feedback between $\mathrm{p} 21$ and reactive oxygen production is necessary for cell senescence. Mol Syst Biol 6:347

46. Kondratskyi A et al (2015) Ion channels in the regulation of apoptosis. Biochim Biophys Acta 1848(10 Pt B):2532-2546

47. Chen $\mathrm{D}$ et al (2014) Inhibition of $\mathrm{Na}^{+} / \mathrm{K}^{+}$-ATPase induces hybrid cell death and enhanced sensitivity to chemotherapy in human glioblastoma cells. BMC Cancer 14:716

48. Pchejetski D et al (2003) Inhibition of $\mathrm{Na}^{+}, \mathrm{K}^{+}$-ATPase by ouabain triggers epithelial cell death indpendently of inversion of the $\left[\mathrm{Na}^{+}\right]_{\mathrm{i}} /\left[\mathrm{K}^{+}\right]_{\mathrm{i}}$ ratio. Biochem Biophys Res Commun 301(3):735-744

49. Olej B et al (1998) Ouabain induces apoptosis in PHA-activated lymphocytes. Biosci Rep 18:1-7

50. Bortner CD et al (2001) Plasma membrane depolarization without repolarization is an early molecular event in anti-Fas-induced apoptosis. J Biol Chem 276(6):4304-4314

51. Ledbetter ML et al (1986) Cooperation between epithelial cells demonstrated by potassium transfer. Am J Physiol 250:306-313

52. Orlov $\mathrm{SN}$ et al (2001) Inhibition of $\mathrm{Na}^{+}, \mathrm{K}^{+}$pump affects nucleic acid synthesis and smooth muscle cell proliferation via elevation of the $\left[\mathrm{Na}^{+}\right]_{\mathrm{i}} /\left[\mathrm{K}^{+}\right]_{\mathrm{i}}$ ratio: possible implication in vascular remodeling. J Hypertens 19:1559-1565
53. Isaev $\mathrm{NK}$ et al (2000) Inhibition of $\mathrm{Na}^{+}, \mathrm{K}^{+}$-ATPase activity in cultured cerebellar granule cells prevents the onset of apoptosis induced by low potassium. Neurosci Lett 283:41-44

54. Zhou X et al (2001) Inhibition of Na, K-ATPase activates PI3 kinase and inhibits apoptosis in LLC-PK1 cells. Biochem Biophys Res Commun 285(1):46-51

55. Wang E (1995) Senescent human fibroblasts resist programmed cell death, and failure to suppress Bcl2 is involved. Cancer Res 55(11):2284-2292

56. Marcotte R et al (2004) Senescent fibroblasts resist apoptosis by downregulating caspase-3. Mech Ageing Dev 125(10-11):777-783

57. Ryu SJ et al (2007) Failure of stress-induced downregulation of Bcl-2 contributes to apoptosis resistance in senescent human diploid fibroblasts. Cell Death Differ 14(5):1020-1028

58. Sõti C et al (2003) Apoptosis, necrosis and cellular senescence: chaperone occupancy as a potential switch. Aging Cell 2(1):39-45

59. Hampel B et al (2004) Differential regulation of apoptotic cell death in senescent human cells. Exp Gerontol 39(11-12):1713-1721

60. Jeon H, Boo YC (2013) Senescent endothelial cells are prone to TNF- $\alpha$-induced cell death due to expression of FAS receptor. Biochem Biophys Res Commun 438(2):277-282

61. Hoffmann J et al (2001) Aging enhances the sensitivity of endothelial cells toward apoptotic stimuli: important role of nitric oxide. Circ Res 89(8):709-715

62. Burton DG, Faragher RG (2015) Cellular senescence: from growth arrest to immunogenic conversion. Age (Dordr) 37(2):27

63. Baker D et al (2016) Naturally occurring p16Ink4a-positive cells shorten healthy lifespan. Nature 530(7589):184-189

64. Paez-Ribes $M$ et al (2019) Targeting senescent cells in translational medicine. EMBO Mol Med 11(12):e10234

65. Soto-Gamez A et al (2019) Regulation of survival networks in senescent cells: from mechanisms to interventions. J Mol Biol 431(15):2629-2643

66. Wang Z et al (2020) Targeting senescent cells and tumor therapy (review). Int J Mol Med 46(5):1603-1610

67. A study to assess the safety and efficacy of a single dose of UBX0101 in patients with osteoarthritis of the knee. https://clini caltrials.gov/ct2/show/NCT04129944?term=NCT04129944\& rank $=1$

68. Garth E (2020) Senolytics research shows longevity progress. https://www.longevity.technology/senolytics-research-showslongevity-progress/

69. Dolgin E (2020) Send in the senolytics. Nat Biotechnol. https:// doi.org/10.1038/s41587-020-00750-1

Publisher's Note Springer Nature remains neutral with regard to jurisdictional claims in published maps and institutional affiliations.

\section{Authors and Affiliations}

\section{Pavel I. Deryabin ${ }^{1} \cdot$ Alla N. Shatrova ${ }^{2} \cdot$ Aleksandra V. Borodkina $^{1} \mathbb{C}$}

1 Mechanisms of Cellular Senescence Group, Institute of Cytology of the Russian Academy of Sciences, Tikhoretsky Ave. 4, 194064 Saint Petersburg, Russia
2 Laboratory of Intracellular Membranes Dynamic, Institute of Cytology of the Russian Academy of Sciences, Tikhoretsky Ave. 4, 194064 Saint Petersburg, Russia 